\title{
SIM PlanetQuest Key Project Precursor Observations to Detect Gas Giant Planets around Young Stars
}

\author{
Angelle Tanner, ${ }^{1}$ Charles Beichman,,${ }^{1}$ Rachel Akeson, ${ }^{1}$ Andrea Ghez, ${ }^{2}$ Konstantin N. Grankin, ${ }^{3}$ William Herbst, ${ }^{4}$ \\ Lynne Hillenbrand, ${ }^{5}$ Marcos Huerta, ${ }^{6}$ Quinn Konopacky, ${ }^{2}$ Stanimir Metchev, ${ }^{2}$ Subhanjoy Mohanty, ${ }^{7}$ L. Prato, ${ }^{8}$ \\ AND Michal SimON ${ }^{9}$ \\ Received 2007 April 23; accepted 2007 June 11; published 2007 July 12
}

\begin{abstract}
We present a review of precursor observing programs for the SIM PlanetQuest Key Project devoted to detecting Jupiter-mass planets around young stars. In order to ensure that the stars in the sample are free of various sources of astrometric noise that might impede the detection of planets, we have initiated programs to collect photometry, high-contrast images, interferometric data, and radial velocities for stars in both the northern and southern hemispheres. We have completed a high-contrast imaging survey of target stars in Taurus and the Pleiades and found no definitive common proper motion companions within 1" (140 AU) of the SIM targets. Our radial velocity surveys have shown that many of the target stars in Sco-Cen are fast rotators, and a few stars in Taurus and the Pleiades may have substellar companions. Interferometric data of a few stars in Taurus show no signs of stellar or substellar companions with separations of 5-50 mas. The photometric survey suggests that approximately half of the stars initially selected for this program are variable to a degree $(1 \sigma>0.1 \mathrm{mag})$ that would degrade the astrometric accuracy achievable for that star. While the precursor programs are still a work in progress, we provide a comprehensive list of all targets and rank them according to their viability as a result of the observations taken to date. The observable that removes by far the most targets from the SIM young stellar object (YSO) program is photometric variability.
\end{abstract}

\section{INTRODUCTION}

The majority of the over 200 planets found to date have been detected using either radial velocity (RV) or transit studies in orbits ranging from less than $0.1 \mathrm{AU}$ out to beyond $5 \mathrm{AU}$, with a wide range of eccentricities and with masses ranging from less than that of Uranus up to many times that of Jupiter (Butler et al. 2006). However, the host stars of these planets are mature main-sequence stars that were chosen based on their having quiescent photospheres for the successful measurement of small Doppler velocities $\left(<10 \mathrm{~m} \mathrm{~s}^{-1}\right)$. Similarly, stellar photospheres must be quiescent at the millimagnitude level for transit detections, since a Jupiter-mass planet transiting a solar-type star reduces the photometric signal by about $1.4 \%$. Since young

\footnotetext{
${ }^{1}$ Michelson Science Center, California Institute of Technology, Pasadena, CA; angelle.tanner@jpl.nasa.gov.

${ }^{2}$ Division of Astronomy, University of California at Los Angeles, Los Angeles, CA.

${ }^{3}$ Astronomical Institute of the Uzbek Academy of Sciences, Tashkent, Uzbekistan.

${ }^{4}$ Astronomy Department, Wesleyan University, Middletown, CT.

${ }^{5}$ California Institute of Technology, Pasadena, CA.

${ }^{6}$ Department of Astronomy, University of Florida, Gainesville, FL.

${ }^{7}$ Center for Astrophysics, Harvard University, Cambridge, MA.

${ }^{8}$ Lowell Observatory, Flagstaff, AZ.

${ }^{9}$ Department of Physics and Astronomy, State University of New York at Stony Brook, Stony Brook, NY.
}

stars often have radial velocity fluctuations or rotationally broadened line widths of at least $500 \mathrm{~m} \mathrm{~s}^{-1}$ and brightness fluctuations of many percents, RV measurements accurate to $<100 \mathrm{~m} \mathrm{~s}^{-1}$ or transit observations cannot be used to detect planets around young stars. ${ }^{10} \mathrm{~A}$ few potentially planetary-mass objects have been detected at 20-100 AU from young host stars $(<10 \mathrm{Myr})$ by direct coronagraphic imaging; e.g., 2MASSW J1207334-393254 (Chauvin et al. 2005) and GQ Lup (Neuhäuser et al. 2005). However, these companions are only inferred to be of planetary mass by comparison to uncertain evolutionary models that predict the brightness of "young Jupiters" as a function of mass and age (Wuchterl \& Tscharnuter 2003; Baraffe et al. 2003; Burrows et al. 1997). Since dynamical determinations of mass are impossible for objects on such distant orbits, it is difficult to be sure that these are planets and not brown dwarfs. Nor is it even clear that the origin of these distant "young Jupiters" is due to same formation processes that created planets found closer in. Multiple fragmentation events (Boss 2001), rather than core accretion in a dense disk (Ida \& Lin 2004), may be responsible for the formation of these distant objects. As a result of the selection biases of the radial velocity, transit, and direct-imaging tech-

\footnotetext{
${ }^{10}$ A number of groups are attempting RV observations in the near-IR, since at these wavelengths it may be possible to improve on these limits and find a few "hot Jupiters" within 0.1 AU.
} 
niques, we know little about the incidence of close-in planets around young stars, leaving us with many questions about the formation and evolution of gas giant planets.

Given the observational limitations and uncertainties that are inherent to radial velocity and direct-imaging surveys, microarcsecond astrometry is a feasible and direct method for estimating the masses of giant planets around stars in young clusters that lie at distances closer than $140 \mathrm{pc}$ (Beichman 2001). Equation (1) gives the astrometric amplitude $\phi$ in units appropriate to the search for planets around young stars:

$$
\phi=35 \frac{140 \mathrm{pc}}{D_{\mathrm{pc}}} \frac{a_{\mathrm{AU}}}{5.2 \mathrm{AU}} \frac{M_{p}}{M_{J}} \frac{M_{\odot}}{M_{\star}} \mu \mathrm{as},
$$

where $\phi$ is the astrometric amplitude in $\mu$ as, $D$ is the distance to the star in parsecs, $a$ is the orbital semimajor axis of the planet in $\mathrm{AU}, M_{p}$ is the planet mass in Jupiter masses, and $M_{\star}$ is the star's mass in solar masses. Thus, a Jupiter orbiting 5.2 AU away from a $0.8 M_{\odot}$ star at a distance of the youngest stellar associations (1-10 Myr), such as Taurus and Chamaeleon $140 \mathrm{pc}$ away, would produce an astrometric amplitude of $44 \mu \mathrm{as}$. At the $25-50 \mathrm{pc}$ distance of the nearest young stars (10-50 Myr), such as members of the $\beta$ Pic and TW Hya moving groups, the same system would have an astrometric amplitude in excess of $100 \mu$ as. Moving a Jupiter into a $1 \mathrm{AU}$ orbit would reduce the signal by a factor of 5.2 , or $50 \mu \mathrm{as}$, for a star at $25 \mathrm{pc}$, and $8 \mu$ as for one in Taurus. Table 1 lists the star formation regions and young moving groups being included in the SIM-YSO survey, a SIM PlanetQuest Key Project aimed at detecting Jupiter-mass planets around young stars. Since SIM will be able to detect astrometric signals with a single-measurement accuracy (SMA) of $4 \mu$ as $(1 \sigma)$ in a fairly quick "narrow angle" (NA) observation, and $11 \mu$ as in a single "wide angle" (WA) observation, a search for gas giants falls well within SIM's capabilities for wide- and narrow-angle astrometry and forms the core of the SIM-YSO program. With SIM's sensitivity, it is reasonable to study stars brighter than $R \sim 12$ mag to a level such that the expected astrometric amplitude of $8 \mu$ as for stars at $140 \mathrm{pc}$ is detected with $2 \sigma$ confidence in each measurement. This astrometric accuracy is appropriate for detecting planets of unknown orbital parameters with a series of approximately 75-100 one-dimensional measurements (Sozzetti et al. 2003; Catanzarite et al. 2006).

Figure 1 shows orbital location (semimajor axis) and $M \sin i$ for over 200 known planets orbiting nearby mature stars. ${ }^{11}$ These planets were found using radial velocity measurements with noise levels as low as $1 \mathrm{~m} \mathrm{~s}^{-1}$. However, for the young stars considered here, the radial velocity measurements will be limited, even in the near-infrared, to $100-500 \mathrm{~m}$ $\mathrm{s}^{-1}$ (or greater), due to rapid rotation, veiling, and photospheric variability. Thus, we plot a RV sensitivity curve for planets

\footnotetext{
${ }^{11}$ Planet information from the Extrasolar Planets Encyclopaedia at http:// exoplanet.eu (J. Schneider, 2007).
}

TABLE 1

SIM-YSO SAMPLE

\begin{tabular}{|c|c|c|c|}
\hline Cluster & $\begin{array}{l}\text { Age } \\
(\mathrm{Myr})\end{array}$ & $\begin{array}{c}\text { Distance } \\
(\mathrm{pc})\end{array}$ & No. of Stars \\
\hline$\beta \mathrm{Pic}$ & 20 & $10-50$ & 16 \\
\hline Chameleon ... & $1-10$ & 140 & 8 \\
\hline$\eta$ Cha $\ldots . . . . . . . . . .$. & $4-7$ & 100 & 2 \\
\hline Horologium ... & 30 & 60 & 12 \\
\hline IC $2391 \ldots$ & 53 & 155 & 12 \\
\hline Ophiuchus $\ldots . . . \ldots . .$. & 2 & 160 & 5 \\
\hline Pleiades........$\ldots$. & 125 & 130 & 14 \\
\hline TW Hydra ........... & 10 & 60 & 15 \\
\hline Taurus-Aureiga ...... & 2 & 140 & 25 \\
\hline Tucanae $. . . . . . . \ldots .$. & 20 & 45 & 20 \\
\hline Upper Sco ........... & $1-10$ & 145 & 49 \\
\hline Sco Cen $\ldots . . . . . . .$. & $1-25$ & 130 & 81 \\
\hline
\end{tabular}

orbiting a $1 M_{\odot}$ star, assuming a limiting accuracy of $100 \mathrm{~m}$ $\mathrm{s}^{-1}$. For comparison, we plot the astrometric sensitivity curve for our SIM-YSO project, where we demand that the minimum detectable planet have an amplitude of at least $8 \mu$ as. The curve is plotted for a $1 M_{\odot}$ star located at the distance of Taurus $(140 \mathrm{pc})$. For planets with periods greater than the nominal mission duration of 5 years, we have degraded the sensitivity as shown schematically in the plot. Finally, we estimate how a coronagraph on a $30 \mathrm{~m}$ telescope or an interferometer with an $85 \mathrm{~m}$ baseline, both operating at $1.6 \mu \mathrm{m}$ and reaching down to the Jovian-mass range at orbital distances of $10 \mathrm{AU}$ or greater, would complement but not replace SIM observations.

In order to maximize the scientific yield of this SIM key project, a significant effort is required to gather information about the target stars prior to the launch of SIM. A careful vetting of the target list is required in order to reject stars that might be problematic due to either the presence of starspots that might induce large astrometric offsets, the presence of circumstellar emission from scattered light, or the presence of visible or spectroscopic companions.

To ensure that we observe astrometrically stable systems, we have initiated a series of precursor observations to check for nearby infrared companions ( $(3)$, radial velocity variations due to unseen companions ( $\$ 4)$, and photometric variability (§ 5). Table 2 summarizes the precursor programs currently being conducted, including the telescopes and the principal investigators. In subsequent sections, we summarize the different programs and give detailed results from a number of them. The SIM-YSO target list will continue to evolve as we add new stars for their scientific interest and remove stars due to one failing or another. In the end, we intend to have a complete sample in both stellar age and mass that will allow for a statistically significant study of planets around young stars.

This paper describes the present status of the SIM-YSO sample, the overall strategy for the precursor vetting program, and detailed results of one specific program, the Palomar AO survey, aimed at identifying companions to the SIM-YSO targets in the Pleiades and Taurus. We summarize briefly the progress 


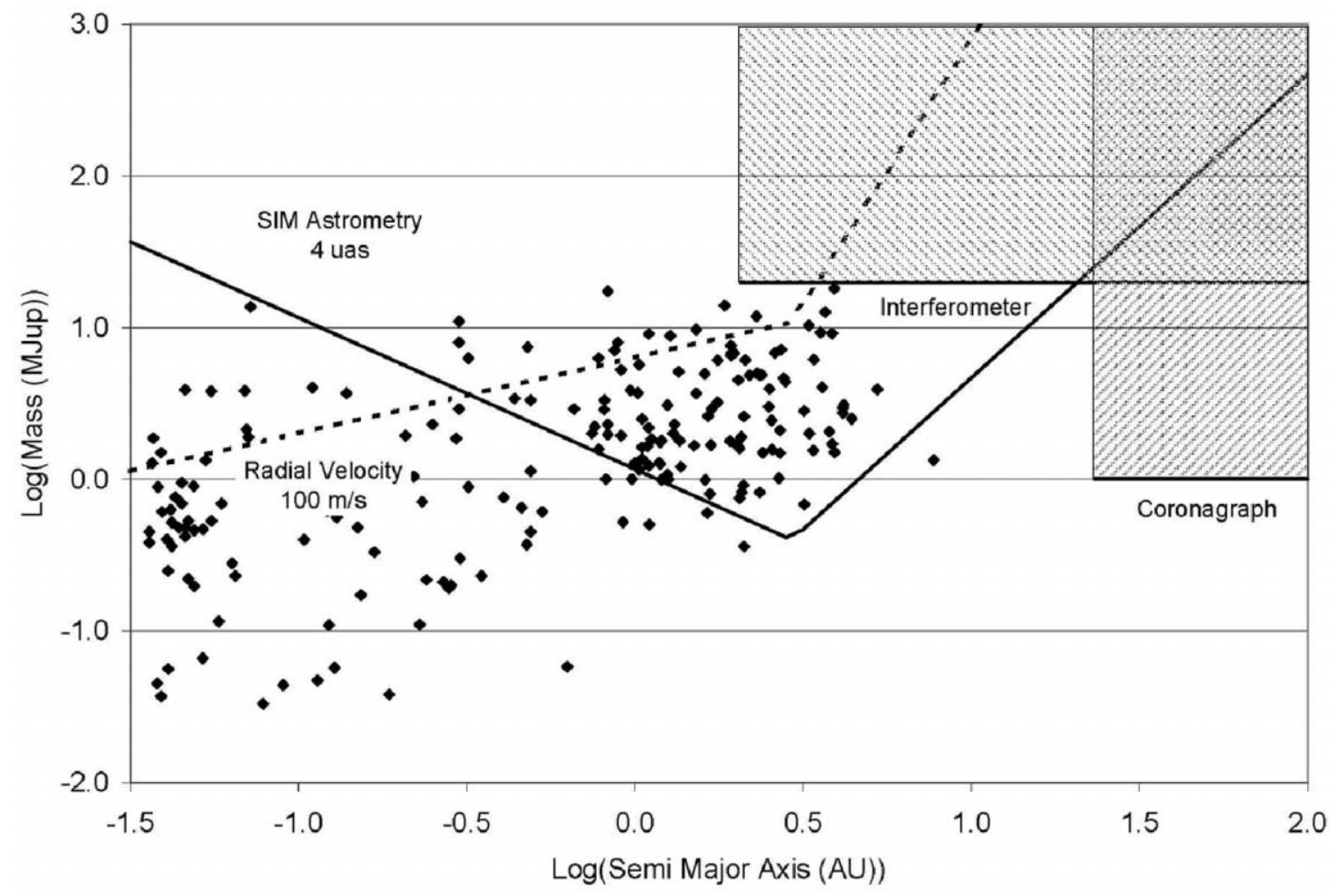

FIG. 1.-Plot of planet mass in $M_{\mathrm{J}}$ vs. semimajor axis of the sensitivities expected from the SIM-YSO survey (solid line), in addition to ground-based coronagraph (labeled), interferometry (labeled), and radial velocity (dashed line) surveys of young stars. Also plotted are the properties of the known radial velocity planets (diamonds). All these sensitivity limits assume a distance of $140 \mathrm{pc}$.

being made on our photometric and radial velocity surveys of both the northern and southern targets, and their implications for the SIM-YSO target sample.

\section{THE SIM-YSO STELLAR SAMPLE OF YOUNG STARS}

In a survey of $\sim 200$ young stars, we expect to find anywhere from 10-20 to 200 planetary systems, depending on whether the 5\%-10\% of stars with known radial velocity planets are representative of the younger planet population, or whether all young stars have planets, only to lose them to inward migration. The youngest stars in the sample (see Table 1) will be located in well-known star-forming regions and will be observed in narrow-angle mode, which is capable of achieving SMAs of $4 \mu$ as. Somewhat older stars, such as those in the $\beta$ Pictoris and TW Hydrae associations, are only $25-50 \mathrm{pc}$ away and can be observed less expensively in a wide-angle mode capable of SMAs of $<11 \mu$ as (Unwin 2005).

We have set our sensitivity threshold to ensure the detection of Jupiter-mass planets in the critical orbital range of 1 to 5 AU. These observations, when combined with the results of the SIM planetary searches of mature stars, will allow us to test theories of planetary formation and early solar system evolution. By searching for planets around pre-main-sequence stars carefully selected to span an age range from 1 to $100 \mathrm{Myr}$, we will learn at what epoch and with what frequency

TABLE 2

PRECURSOR Programs

\begin{tabular}{llll}
\hline \hline \multicolumn{1}{c}{ Program } & \multicolumn{1}{c}{ Telescope } & \multicolumn{1}{c}{ PI } \\
\hline AO imaging (north) $\ldots \ldots \ldots \ldots \ldots \ldots \ldots$ & Palomar & A. Tanner \\
AO imaging (south) $\ldots \ldots \ldots \ldots \ldots \ldots \ldots$ & VLT & C. Dumas \\
Speckle imaging (north) $\ldots \ldots \ldots \ldots \ldots \ldots$ & Keck & A. Ghez, Q. Konopacky \\
Interferometric visibilities (north) $\ldots \ldots$ & Keck & R. Akeson \\
RV survey (north) $\ldots \ldots \ldots \ldots \ldots \ldots \ldots \ldots$ & McDonald & L. Prato \\
RV survey (south) $\ldots \ldots \ldots \ldots \ldots \ldots \ldots \ldots$ & CTIO/Magellan & S. Mohanty \\
Photometry (north) $\ldots \ldots \ldots \ldots \ldots \ldots \ldots$ & Maidanak & K. Grankin \\
Photometry (south) & $\ldots \ldots \ldots \ldots \ldots \ldots \ldots$ & SMARTS & M. Simon \\
\hline
\end{tabular}


giant planets are found at the water-ice "snow line," where they are expected to form (Pollack et al. 1996). This will provide insight into the physical mechanisms by which planets form and migrate from their place of birth, as well as their survival rate. With these observations in hand, we will provide data, for the first time, on such important questions as: What processes affect the formation and dynamical evolution of planets? When and where do planets form? What is the initial mass distribution of planetary systems around young stars? How might planets be destroyed? What is the origin of the eccentricity of planetary orbits? What is the origin of the apparent dearth of companion objects between planets and brown dwarfs seen in mature stars? How might the formation and migration of gas giant planets affect the formation of terrestrial planets?

Our observational strategy is a compromise between the desire to extend the planetary-mass function as low as possible and the essential need to build up sufficient statistics on planetary occurrence. About half of the sample will be used to address the "where" and "when" of planet formation. We will study classical T Tauri stars (CTTSs) that have massive accretion disks, as well as postaccretion, weak-lined T Tauri stars (WTTSs). Preliminary estimates suggest the sample will consist of $\sim 30 \%$ CTTSs and $~ 70 \%$ WTTSs, driven in part by the difficulty of making accurate astrometric measurements toward objects with strong variability or prominent disks. The extent to which this distribution of CTTSs and WTTSs survives the screening programs for photometric and dynamic stability is addressed in $\S 6$. The second half of the sample will be drawn from the closest young clusters with ages starting around $5 \mathrm{Myr}$ to the $10 \mathrm{Myr}$ point thought to mark the end of prominent disks, and ending around the $100 \mathrm{Myr}$ age, at which theory suggests that the properties of young planetary systems should become indistinguishable from those of mature stars. The properties of the planetary systems found around stars in these later age bins will be used to address the effects of dynamical evolution and planet destruction (Lin 2001).

We have adopted the following criteria in developing our initial list of candidates: (1) stellar mass between 0.2 and $2.0 M_{\odot}$, (2) $R<12 \mathrm{mag}$ for reasonable integration times, (3) distance less than $140 \mathrm{pc}$ to ensure an astrometric signal greater than $6 \mu$ as, (4) no companions within 2" and $100 \mathrm{AU}$ for instrumental and scientific considerations, respectively, (5) no nebulosity to confuse the astrometric measurements, (6) variability $\Delta R<0.1 \mathrm{mag}$, and (7) a spread of ages between 1 and 100 Myr to encompass the expected time period of planetdisk and early planet-planet interactions. With proper selection, the effect of various astrophysical disturbances can be kept to less than the few $\mu$ as needed to detect Jupiter-mass planets at $\sim 50-140 \mathrm{pc}$.

The initial SIM-YSO sample (see Table 3) consists of stars in the well-known star-forming regions and close associations. Figure 2 shows histograms of the properties of the stars in the sample, including distance, $V$ magnitude, and age. The stars included in the initial sample have been screened for binarity in either imaging (Stauffer et al. 1998; Lowrance et al. 2005) or spectroscopic surveys (White \& Ghez 2001; Mathieu et al. 1997; Steffen et al. 2001).

\section{HIGH-CONTRAST DIRECT IMAGING}

We begin by presenting the results of a companion survey for the SIM-YSO targets in our Taurus and Pleiades samples (see Table 4). Companions within the 1.5" field of view of the SIM interferometer that have magnitudes within $\Delta V \sim 4$ mag (M. Shao 2007, private communication) could cause a bias in the position of the fringe used to make the astrometric measurements. In addition, a massive unknown stellar companion will induce astrometric perturbations, complicating the astrometric solution for a planet around the primary star. To look for common proper motion companions to the SIM-YSO stars, we have conducted an adaptive optics (AO) coronagraphic imaging survey around 31 stars in the Taurus $(2 \mathrm{Myr}, 140 \mathrm{pc}$; Kenyon et al. 1994) and Pleiades (120 Myr, 135 pc; Stauffer et al. 1998; Pan et al. 2004) clusters with the Palomar Adaptive Optics (PALAO) system and its near-infrared Palomar High Angular Resolution Observer (PHARO) camera on the Hale 200 inch $(5 \mathrm{~m})$ telescope (Hayward et al. 2001). These data will reveal the presence of stellar and brown dwarf companions located between $\sim 50$ and $1000 \mathrm{AU}$, and in the case of the youngest stars in these systems ( $\sim 2 \mathrm{Myr}$ ), will be sensitive to hot, young planets with masses in the range of $10 M_{\mathrm{J}}-20 M_{\mathrm{J}}$ (Burrows et al. 1997; Baraffe et al. 2003).

In the process of searching for unseen companions around these stars, we are also addressing planet formation issues. By investigating whether the "brown dwarf desert" observed for separations of $<5 \mathrm{AU}$ around main-sequence stars (Marcy \& Butler 2000) also exists at larger separations for young stars, we can test whether brown dwarfs are formed at this separation and subsequently migrate inward and are destroyed by falling onto the star. In this case, we might find that $\mathrm{T}$ Tauri stars have a larger population of brown dwarfs than main-sequence stars at these separations.

\subsection{Data Reduction and Analysis}

The Palomar observations were obtained over three observing runs (2003 October 23, 2003 December 4-6, and 2005 November 12-14), with good $\left(0.2^{\prime \prime}\right)$ to moderate $\left(0.5^{\prime \prime}\right)$ seeing throughout the nights. The PHARO-PALAO camera has a pixel scale of 25 mas pixel $^{-1}$ and a field of view of $25^{\prime \prime}$. Each target was observed with the $0.97^{\prime \prime}$ diameter occulting spot placed over the star, with integration times of $60 \mathrm{~s}$ each and multiple (10-20) images collected per target. Sky images were also taken adjacent to each set of target images by offsetting $30^{\prime \prime}$ from the target in the four cardinal directions. For flux calibration, observations of the target stars were taken with the star offset from the coronagraph in a five-point dither pattern to allow for adequate sky subtraction. To improve observing efficiency, those stars with similar magnitudes and colors were 
TABLE 3

SIM-YSO SAMPLE

\begin{tabular}{|c|c|c|c|c|c|c|c|c|c|}
\hline Name & Cluster & $\begin{array}{l}\text { Distance } \\
\quad(\mathrm{pc})\end{array}$ & Spectral Type & $\begin{array}{l}\text { Age } \\
(\mathrm{Myr})\end{array}$ & $\begin{array}{c}\text { Star Mass }{ }^{\mathrm{a}} \\
\left(M_{\odot}\right)\end{array}$ & $\begin{array}{c}\text { Signal } \\
(\mu \mathrm{as})\end{array}$ & T Tauri Class & $\begin{array}{c}V \\
(\mathrm{mag})\end{array}$ & $\begin{array}{c}\text { 2MASS } K_{s} \\
(\mathrm{mag})\end{array}$ \\
\hline \multicolumn{10}{|c|}{ Low-Variability Targets } \\
\hline PreibZinn $9964^{\mathrm{b}}$ & U Sco & 145 & M1 & 0.1 & 0.3 & 23.68 & $\ldots$ & $\ldots$ & 8.36 \\
\hline 51 Eri $\ldots \ldots \ldots \ldots \ldots \ldots \ldots$ & $\beta \mathrm{Pic}$ & 29.8 & F0 V & 20 & 1.5 & 21.51 & $\ldots$ & 5.22 & 4.54 \\
\hline PreibZinn $9914 \quad \ldots \ldots \ldots \ldots$ & U Sco & 145 & M0.5 & 0.5 & 0.3 & 19.50 & $\ldots$ & $\ldots$ & 9.09 \\
\hline RECX $10 \ldots \ldots \ldots$ & $\eta$ Cha & 100 & K6 & 7 & 0.6 & 17.48 & WTT & 12.53 & 8.73 \\
\hline PreibZinn $9928 \quad \ldots \ldots \ldots \ldots$ & U Sco & 145 & M0 & 1 & 0.4 & 15.79 & $\ldots$ & $\ldots$ & 8.80 \\
\hline PreibZinn $9913 \quad \ldots \ldots \ldots \ldots$ & U Sco & 145 & M0 & 1.2 & 0.4 & 15.07 & $\ldots$ & $\ldots$ & 8.88 \\
\hline PreibZinn 9919 & U Sco & 145 & K6 & 0.7 & 0.5 & 12.51 & $\ldots$ & $\ldots$ & 8.11 \\
\hline PreibZinn $9974 \quad \ldots .$. & U Sco & 145 & K4 & 0.5 & 0.6 & 10.36 & $\ldots$ & $\ldots$ & 8.46 \\
\hline PreibZinn $9936 \quad \ldots \ldots \ldots \ldots$ & U Sco & 145 & K7 & 2.7 & 0.7 & 10.20 & $\ldots$ & $\ldots$ & 9.12 \\
\hline PreibZinn $9967 \quad \ldots \ldots \ldots \ldots$ & U Sco & 145 & K5 & 1.1 & 0.7 & 10.05 & $\ldots$ & $\ldots$ & 8.56 \\
\hline PreibZinn $9969 \quad \ldots \ldots \ldots \ldots$ & U Sco & 145 & K5 & 1.8 & 0.7 & 9.47 & $\ldots$ & $\ldots$ & 8.56 \\
\hline HII $489 \ldots \ldots$. & Pleiades & 130 & F8 & 125 & $\ldots$ & 9.25 & $\ldots$ & 10.38 & 8.87 \\
\hline HII 1794 & Pleiades & 130 & F8 & 125 & $\ldots$ & 9.25 & $\ldots$ & 10.20 & 8.89 \\
\hline HII $2366 \quad \ldots \ldots \ldots \ldots$ & Pleiades & 130 & $\mathrm{G} 2$ & 125 & $\ldots$ & 9.25 & $\ldots$ & 11.53 & 9.55 \\
\hline TYC $8283-2795-1 \quad \ldots \ldots \ldots$ & Sco-Cen (UCL) & 130 & & $\ldots$ & $\ldots$ & 9.25 & WTT & 10.79 & 8.98 \\
\hline PreibZinn 9950 ................. & U Sco & 145 & K5 & 2.5 & 0.8 & 8.84 & $\ldots$ & $\ldots$ & 8.90 \\
\hline L1551-55 „............... & Tau Aur & 140 & K7 & 2 & $\ldots$ & 8.59 & $\ldots$ & 13.22 & 9.31 \\
\hline PreibZinn $9939 \quad \ldots \ldots \ldots \ldots$ & U Sco & 145 & K4 & 2.2 & 0.8 & 8.29 & $\ldots$ & $\ldots$ & 8.73 \\
\hline HII $1124 \ldots \ldots$ & Pleiades & 130 & $\mathrm{~K} 3 \mathrm{~V}$ & 125 & 1.0 & 7.79 & $\ldots$ & 12.12 & 9.86 \\
\hline PreibZinn $9958 \quad \ldots \ldots \ldots \ldots$ & U Sco & 145 & $\mathrm{~K} 2$ & 1.2 & 0.9 & 7.62 & $\ldots$ & $\ldots$ & 8.43 \\
\hline HII $1095 \quad \ldots \ldots \ldots \ldots \ldots \ldots$ & Pleiades & 130 & $\mathrm{~K} 0 \mathrm{~V}$ & 125 & 1.0 & 7.40 & $\ldots$ & 11.92 & 9.67 \\
\hline HII 1309 & Pleiades & 130 & F6 V & 125 & 1.0 & 7.40 & $\ldots$ & 9.58 & 8.28 \\
\hline HII $1613 \ldots$ & Pleiades & 130 & F8 V & 125 & 1.0 & 7.40 & $\ldots$ & 9.87 & 8.57 \\
\hline HII $1797 \ldots$ & Pleiades & 130 & F9 V & 125 & 1.0 & 7.40 & $\ldots$ & 10.09 & 15.04 \\
\hline HII 1856 & Pleiades & 130 & F8 V & 125 & 1.0 & 7.40 & $\ldots$ & 10.20 & 8.66 \\
\hline TYC $8654-1115-1 \quad \ldots \ldots \ldots$. & Sco-Cen (LCC) & 130 & & 24 & 1.0 & 7.40 & WTT & 10.21 & 8.13 \\
\hline TYC $8295-1530-1 \quad \ldots \ldots \ldots$ & Sco-Cen (UCL) & 130 & G5 & 21 & 1.0 & 7.40 & WTT & 10.98 & 8.90 \\
\hline PreibZinn 9945 ...... & U Sco & 145 & $\mathrm{~K} 2$ & 1.2 & 0.9 & 7.37 & $\ldots$ & 11.17 & 8.04 \\
\hline HD $141569 \ldots \ldots \ldots \ldots \ldots$ & None & 35 & B9.5e & 5 & 4.0 & 6.87 & $\ldots$ & 7.11 & 6.82 \\
\hline TYC $8667-283-1 \ldots \ldots \ldots \ldots$ & Sco-Cen (LCC) & 130 & G3/G5 V & 23 & 1.1 & 6.72 & WTT & 9.31 & 7.62 \\
\hline TYC 7783-1908-1 ........ & Sco-Cen (LCC) & 130 & G8 IV: & 18 & 1.1 & 6.72 & WTT & 9.82 & 7.51 \\
\hline TYC 8258-1878-1 ........ & Sco-Cen (LCC) & 130 & & 15 & 1.1 & 6.72 & WTT & 10.62 & 8.27 \\
\hline TYC 9244-814-1 .......... & Sco-Cen (LCC) & 130 & G3/G5 III & 22 & 1.1 & 6.72 & WTT & 10.21 & 8.40 \\
\hline TYC 8270-2015-1 $\ldots \ldots \ldots$ & Sco-Cen (UCL) & 130 & & 17 & 1.1 & 6.72 & WTT & 10.91 & 8.69 \\
\hline TYC 7851-1-1 $\ldots \ldots \ldots \ldots$ & Sco-Cen (UCL) & 130 & G9 & 17 & 1.1 & 6.72 & WTT & 10.63 & 8.36 \\
\hline TYC $7353-2640-1 \quad \ldots \ldots \ldots$ & Sco-Cen (UCL) & 130 & & 18 & 1.1 & 6.72 & WTT & 10.72 & 8.67 \\
\hline CHXR $8 \ldots \ldots \ldots \ldots \ldots \ldots$ & Cham & 140 & G0 & 100 & 1.1 & 6.24 & WTT & 11.45 & 9.73 \\
\hline HII $430 \ldots \ldots \ldots \ldots \ldots \ldots$ & Pleiades & 130 & G8 V & 125 & 1.2 & 6.16 & $\ldots$ & 11.40 & 9.47 \\
\hline HII 1032 ................. & Pleiades & 130 & $\mathrm{~A} 2$ & 125 & 1.2 & 6.16 & $\ldots$ & 11.10 & 9.16 \\
\hline HII $1136 \quad \ldots \ldots \ldots \ldots \ldots \ldots$ & Pleiades & 130 & G7 V & 125 & 1.2 & 6.16 & $\ldots$ & 12.02 & 12.14 \\
\hline HII $1275 \ldots \ldots \ldots \ldots \ldots$ & Pleiades & 130 & K0 V & 125 & 1.2 & 6.16 & $\ldots$ & 11.47 & 9.53 \\
\hline TYC $8646-166-1 \ldots \ldots \ldots \ldots$ & Sco-Cen (LCC) & 130 & & 11 & 1.2 & 6.16 & WTT & 10.50 & 8.18 \\
\hline TYC 8636-2515-1 ........ & Sco-Cen (LCC) & 130 & & 11 & 1.2 & 6.16 & WTT & 10.58 & 8.12 \\
\hline TYC 8633-508-1 .......... & Sco-Cen (LCC) & 130 & K2 IV:+ & 16 & 1.2 & 6.16 & WTT & 9.41 & 7.65 \\
\hline TYC 9245-617-1 .......... & Sco-Cen (LCC) & 130 & & 10 & 1.2 & 6.16 & WTT & 10.01 & 7.55 \\
\hline TYC 8652-1791-1 ........ & Sco-Cen (LCC) & 130 & F6/F7 & 16 & 1.2 & 6.16 & WTT & 10.35 & 8.48 \\
\hline TYC 8259-689-1 .......... & Sco-Cen (LCC) & 130 & & 14 & 1.2 & 6.16 & WTT & 10.48 & 8.10 \\
\hline TYC 8248-539-1 ........... & Sco-Cen (LCC) & 130 & $\mathrm{G} 1 / \mathrm{G} 2$ & 26 & 1.2 & 6.16 & WTT & 10.10 & 8.54 \\
\hline HD $120411 \ldots \ldots \ldots \ldots \ldots$ & Sco-Cen (UCL) & 130 & $\mathrm{G} 1 \mathrm{~V}$ & 20 & 1.2 & 6.16 & WTT? & 9.79 & 8.16 \\
\hline V1009 Cen .................. & Sco-Cen (UCL) & 130 & $\mathrm{G} 8 / \mathrm{K} 0 \mathrm{~V}$ & 13 & 1.2 & 6.16 & WTT? & 10.18 & 7.95 \\
\hline TYC $7310-2431-1 \quad \ldots \ldots \ldots$ & Sco-Cen (UCL) & 130 & G5 & 16 & 1.2 & 6.16 & WTT & 10.36 & 8.28 \\
\hline TYC 8297-1613-1 ......... & Sco-Cen (UCL) & 130 & & 17 & 1.2 & 6.16 & WTT & 10.22 & 8.51 \\
\hline TYC $7822-158-1 \ldots \ldots \ldots \ldots$ & Sco-Cen (UCL) & 130 & $\mathrm{~K} 1$ & 13 & 1.2 & 6.16 & WTT & 11.11 & 8.51 \\
\hline TYC 7848-1659-1 $\ldots \ldots \ldots$ & Sco-Cen (UCL) & 130 & G5 & 15 & 1.2 & 6.16 & WTT & 10.36 & 8.21 \\
\hline HD $140421 \ldots \ldots \ldots \ldots \ldots$ & Sco-Cen (UCL) & 130 & $\mathrm{G} 1 \mathrm{~V}$ & 17 & 1.2 & 6.16 & WTT? & 9.46 & 7.87 \\
\hline TYC 8317-551-1 .......... & Sco-Cen (UCL) & 130 & G0 & 13 & 1.2 & 6.16 & WTT & 10.29 & 8.27 \\
\hline TYC 7333-1260-1 $\ldots \ldots \ldots$ & Sco-Cen (UCL) & 130 & $\mathrm{G} 1 / \mathrm{G} 2 \mathrm{~V}$ & 18 & 1.2 & 6.16 & WTT & 9.58 & 8.07 \\
\hline PreibZinn $9922 \quad \ldots \ldots \ldots \ldots$ & U Sco & 145 & G0 & 18 & 1.1 & 6.03 & $\ldots$ & $\ldots$ & 8.77 \\
\hline TYC 9231-1566-1 ........ & Sco-Cen (LCC) & 130 & G3 IV & 12 & 1.3 & 5.69 & WTT & 9.23 & 7.18 \\
\hline TYC $8263-2453-1 \quad \ldots \ldots \ldots$. & Sco-Cen (UCL) & 130 & F8/G0 V & 14 & 1.3 & 5.69 & WTT & 9.69 & 7.94 \\
\hline
\end{tabular}


TANNER ET AL.

TABLE 3 (Continued)

\begin{tabular}{|c|c|c|c|c|c|c|c|c|c|}
\hline Name & Cluster & $\begin{array}{l}\text { Distance } \\
\text { (pc) }\end{array}$ & Spectral Type & $\begin{array}{l}\text { Age } \\
(\mathrm{Myr})\end{array}$ & $\begin{array}{c}\text { Star Mass }^{\mathrm{a}} \\
\left(M_{\odot}\right)\end{array}$ & $\begin{array}{l}\text { Signal } \\
\text { ( } \mu \text { as })\end{array}$ & $\mathrm{T}$ Tauri Class & $\begin{array}{c}V \\
(\mathrm{mag})\end{array}$ & $\begin{array}{c}\text { 2MASS } K_{s} \\
(\mathrm{mag})\end{array}$ \\
\hline TYC 7813-224-1 .......... & Sco-Cen (UCL) & 130 & & 14 & 1.3 & 5.69 & WTT & 10.55 & 8.39 \\
\hline TYC 8683-242-1 .......... & Sco-Cen (UCL) & 130 & & 8 & 1.3 & 5.69 & WTT & 10.80 & 8.30 \\
\hline TYC 7828-2913-1 ........ & Sco-Cen (UCL) & 130 & & 5 & 1.3 & 5.69 & WTT & 11.02 & 8.29 \\
\hline TYC 7310-503-1 .......... & Sco-Cen (UCL) & 130 & $\mathrm{~K} 3$ & 2 & 1.3 & 5.69 & WTT & 10.88 & 7.87 \\
\hline TYC 7845-1174-1 .. & Sco-Cen (UCL) & 130 & $\mathrm{~K} 1$ & 3 & 1.3 & 5.69 & WTT & 10.61 & 7.93 \\
\hline TYC 7349-2191-1 ........ & Sco-Cen (UCL) & 130 & & 1 & 1.3 & 5.69 & WTT & 11.09 & 8.29 \\
\hline PreibZinn $9975 \ldots$ & U Sco & 145 & G9 & 1 & 1.2 & 5.62 & $\ldots$ & 10.50 & 7.43 \\
\hline HII $1514 \ldots \ldots \ldots$. & Pleiades & 130 & G5 V & 125 & 1.4 & 5.28 & $\ldots$ & 10.48 & 8.95 \\
\hline HD $140374 \ldots \ldots \ldots$ & Sco-Cen (UCL) & 130 & G8 V & 8 & 1.4 & 5.28 & WTT? & 9.69 & 7.80 \\
\hline CHXR $6 \ldots \ldots \ldots \ldots \ldots \ldots$ & Cham & 140 & $\mathrm{~K} 2$ & 1 & 1.3 & 5.28 & CTT & 11.22 & 7.31 \\
\hline PreibZinn 9979 ...... & $\mathrm{USco}$ & 145 & G5 & 9 & 1.3 & 5.18 & $\cdots$ & $\cdots$ & 8.69 \\
\hline \multicolumn{10}{|c|}{ High-Variability Targets } \\
\hline PreibZinn 9980 & U Sco & 145 & M1 & 0.3 & 0.3 & 22.87 & $\ldots$ & $\ldots$ & 7.91 \\
\hline PreibZinn $9940 \quad \ldots \ldots \ldots \ldots$ & U Sco & 145 & M2 & 0.4 & 0.3 & 22.10 & $\ldots$ & $\ldots$ & 8.61 \\
\hline PreibZinn 9970 .......... & U Sco & 145 & M1 & 0.5 & 0.3 & 20.72 & $\ldots$ & $\ldots$ & 8.82 \\
\hline PreibZinn $9955 \quad \ldots \ldots \ldots \ldots$ & U Sco & 145 & & 0.5 & 0.3 & 20.09 & $\ldots$ & $\ldots$ & 8.10 \\
\hline PreibZinn $9933 \quad \ldots \ldots \ldots \ldots$ & U Sco & 145 & M1 & 0.8 & 0.4 & 18.95 & $\ldots$ & $\ldots$ & 8.84 \\
\hline RECX $4 \ldots \ldots \ldots \ldots \ldots$ & $\eta$ Cha & 100 & K7 & 4 & 0.5 & 18.49 & WTT & 12.79 & 8.62 \\
\hline PreibZinn $9921 \ldots$. & U Sco & 145 & M1 & 0.8 & 0.4 & 18.42 & $\ldots$ & $\ldots$ & 8.63 \\
\hline PreibZinn $993 \ldots \ldots \ldots \ldots$ & U Sco & 145 & M1 & 1 & 0.4 & 17.45 & $\ldots$ & $\ldots$ & 9.08 \\
\hline PreibZinn 996 ............ & U Sco & 145 & M0 & 1 & 0.4 & 15.42 & $\ldots$ & $\ldots$ & 8.98 \\
\hline PreibZinn 9963 .......... & U Sco & 145 & M0 & 1.8 & 0.5 & 14.74 & $\ldots$ & $\ldots$ & 8.44 \\
\hline DM Tau $\ldots \ldots \ldots \ldots \ldots \ldots$ & $\tau$ Aur & 140 & K5 V:e & 2 & 0.5 & 14.61 & CTT & 13.78 & 9.52 \\
\hline PreibZinn 9959 ............ & U Sco & 145 & M0 & 2 & 0.5 & 14.11 & $\ldots$ & $\ldots$ & 8.91 \\
\hline PreibZinn $9962 \ldots$ & U Sco & 145 & M0 & 2 & 0.5 & 14.11 & $\ldots$ & $\ldots$ & 8.92 \\
\hline CHXR $29 \ldots \ldots$. & Cham & 140 & A0pshe & $\ldots$ & 0.5 & 13.74 & CTT & 8.44 & 5.94 \\
\hline CHXR $18 \mathrm{~N} \quad \ldots \ldots \ldots \ldots \ldots$ & Cham & 140 & K1 & $\ldots$ & 0.5 & 13.74 & WTT & 12.05 & 7.77 \\
\hline CHXR 68A $\ldots \ldots \ldots \ldots \ldots$ & Cham & 140 & & $\ldots$ & 0.5 & 13.74 & WTT & 13.37 & 8.87 \\
\hline T Cha $\ldots \ldots \ldots \ldots \ldots \ldots \ldots$ & Cham & 140 & F5 & $\ldots$ & 0.5 & 13.74 & CTT & 11.86 & 6.95 \\
\hline PreibZinn $9911 \quad \ldots \ldots \ldots \ldots$ & U Sco & 145 & K7 & 0.8 & 0.5 & 13.26 & $\ldots$ & $\ldots$ & 8.37 \\
\hline PreibZinn $9916 \quad \ldots \ldots \ldots \ldots$ & U Sco & 145 & M0 & 2.9 & 0.5 & 13.26 & $\ldots$ & $\ldots$ & 9.27 \\
\hline DN Tau $\ldots \ldots \ldots \ldots \ldots \ldots$ & $\tau$ Aur & 140 & K6 V:e & 0.46 & 0.6 & 12.26 & CTT & 12.53 & 8.02 \\
\hline IP Tau $\ldots \ldots \ldots \ldots \ldots \ldots \ldots$ & $\tau$ Aur & 140 & M0: Ve & 2 & 0.6 & 11.84 & CTT & 13.04 & 8.35 \\
\hline PreibZinn 9926 ........... & U Sco & 145 & K7 & 1.8 & 0.6 & 11.05 & $\ldots$ & $\ldots$ & 8.92 \\
\hline DG Tau $\ldots \ldots \ldots \ldots \ldots \ldots$ & $\tau$ Aur & 140 & G V:e & 2 & 0.7 & 10.57 & CTT & $\ldots$ & 6.99 \\
\hline PreibZinn $9961 \quad \ldots \ldots \ldots \ldots$ & $\mathrm{USco}$ & 145 & K5 & 1.8 & 0.7 & 9.47 & $\ldots$ & $\ldots$ & 8.62 \\
\hline UY Aur $\ldots \ldots \ldots \ldots \ldots \ldots$ & $\tau$ Aur & 140 & G5 V:e & 2 & 0.7 & 9.28 & CTT & 12.40 & 7.24 \\
\hline BP Tau $\ldots \ldots \ldots \ldots \ldots \ldots$ & $\tau$ Aur & 140 & K5 V:e & 0.6 & 0.8 & 9.16 & CTT & 11.96 & 7.74 \\
\hline GK Tau $\ldots \ldots \ldots \ldots \ldots \ldots \ldots$ & $\tau$ Aur & 140 & & 2 & 0.8 & 9.16 & CTT & 12.50 & 7.47 \\
\hline AA Tau $\ldots \ldots \ldots \ldots \ldots \ldots \ldots$ & $\tau$ Aur & 140 & M0 V:e & 2 & 0.8 & 9.04 & CTT & 12.82 & 8.05 \\
\hline HQ Tau $\ldots \ldots \ldots \ldots \ldots \ldots \ldots$ & $\tau$ Aur & 140 & & 0.69 & 0.8 & 9.04 & CTT & $\ldots$ & 7.14 \\
\hline IW Tau .... & $\tau$ Aur & 140 & $\mathrm{~K} 7 \mathrm{~V}$ & 2 & 0.8 & 9.04 & WTT & 12.51 & 8.28 \\
\hline DR Tau $\ldots \ldots \ldots \ldots \ldots \ldots$ & $\tau$ Aur & 140 & K4 V:e & 2 & 0.8 & 9.04 & CTT & 13.60 & 6.87 \\
\hline V830 Tau ................. & $\tau$ Aur & 140 & K7 & 2 & 0.8 & 8.92 & WTT & 12.21 & 8.42 \\
\hline DL Tau $\ldots \ldots \ldots \ldots \ldots \ldots \ldots$ & $\tau$ Aur & 140 & G V:e & 2 & 0.8 & 8.92 & CTT & 13.55 & 7.96 \\
\hline L1551-51 $\ldots \ldots \ldots \ldots \ldots \ldots$ & $\tau$ Aur & 140 & K7 & 2 & $\ldots$ & 8.59 & $\ldots$ & 12.06 & 8.85 \\
\hline CI Tau $\ldots \ldots \ldots \ldots \ldots \ldots \ldots$ & $\tau$ Aur & 140 & G V:e & 2 & $\ldots$ & 8.59 & $\ldots$ & 12.99 & 7.79 \\
\hline V836 Tau ........... & $\tau$ Aur & 140 & $\mathrm{~K} 7 \mathrm{~V}$ & 2.1 & 0.8 & 8.59 & WTT & 13.13 & 8.60 \\
\hline PreibZinn $9918 \quad \ldots \ldots \ldots \ldots$ & U Sco & 145 & $\mathrm{~K} 3$ & 1 & 0.8 & 8.50 & $\ldots$ & $\ldots$ & 8.33 \\
\hline PreibZinn 9942 .......... & U Sco & 145 & $\mathrm{~K} 5$ & 2.9 & 0.8 & 8.50 & $\ldots$ & $\ldots$ & 8.37 \\
\hline PreibZinn 9984 ........... & U Sco & 145 & K3 & 1 & 0.8 & 8.50 & $\ldots$ & $\ldots$ & 8.93 \\
\hline TYC 8648-446-1 .......... & Sco-Cen (LCC) & 130 & & 19 & 0.9 & 8.22 & WTT & 11.18 & 8.79 \\
\hline PreibZinn 9968 .......... & U Sco & 145 & $\mathrm{~K} 2$ & 1 & 0.8 & 7.89 & $\ldots$ & 11.65 & 7.69 \\
\hline SR 4/V2058 Oph $\ldots \ldots \ldots \ldots$ & Ophiuchus & 160 & $\mathrm{~K} 5 \mathrm{e}$ & 2 & $\ldots$ & 7.51 & $\ldots$ & 13.60 & 7.52 \\
\hline DoAr21 Oph $\ldots \ldots \ldots \ldots \ldots$ & Ophiuchus & 160 & $\mathrm{~B} 2 \mathrm{~V}$ & 2 & $\ldots$ & 7.51 & $\ldots$ & 13.82 & 6.23 \\
\hline Haro 1-16 Oph $\ldots \ldots \ldots \ldots \ldots$ & Ophiuchus & 160 & $\mathrm{~K} 3$ & 2 & $\ldots$ & 7.51 & $\ldots$ & 12.59 & 7.61 \\
\hline V1121 Oph $\ldots \ldots \ldots \ldots \ldots \ldots$ & Ophiuchus & 160 & K5 & 2 & $\ldots$ & 7.51 & $\ldots$ & 11.25 & 6.96 \\
\hline V966 Cen $\ldots \ldots \ldots \ldots \ldots \ldots$ & Sco-Cen (LCC) & 130 & $\mathrm{~K} 1: \mathrm{V}:+$ & $\ldots$ & 1.0 & 7.40 & WTT? & 9.76 & 8.08 \\
\hline TYC 7319-749-1 .......... & Sco-Cen (UCL) & 130 & K0 & 20 & 1.0 & 7.40 & WTT & 10.59 & 8.34 \\
\hline GI Tau $\ldots \ldots \ldots \ldots \ldots \ldots \ldots$ & $\tau$ Aur & 140 & K5e & 2 & 0.9 & 7.39 & CTT & 13.50 & 7.89 \\
\hline PreibZinn $991 \ldots \ldots \ldots \ldots$ & U Sco & 145 & $\mathrm{~K} 3$ & 3 & 0.9 & 7.37 & $\ldots$ & $\ldots$ & 9.43 \\
\hline PreibZinn 9937 .......... & U Sco & 145 & $\mathrm{~K} 4$ & 4 & 0.9 & 7.37 & $\ldots$ & $\ldots$ & 8.93 \\
\hline
\end{tabular}


TABLE 3 (Continued)

\begin{tabular}{|c|c|c|c|c|c|c|c|c|c|}
\hline Name & Cluster & $\begin{array}{l}\text { Distance } \\
(\mathrm{pc})\end{array}$ & Spectral Type & $\begin{array}{l}\mathrm{Age} \\
(\mathrm{Myr})\end{array}$ & $\begin{array}{l}\text { Star Mass } \mathrm{a}^{\mathrm{a}} \\
\left(M_{\odot}\right)\end{array}$ & $\begin{array}{l}\text { Signal } \\
(\mu \text { as })\end{array}$ & T Tauri Class & $\begin{array}{c}V \\
(\mathrm{mag})\end{array}$ & $\begin{array}{c}\text { 2MASS } K_{s} \\
(\mathrm{mag})\end{array}$ \\
\hline PreibZinn 9976 & U Sco & 145 & $\mathrm{~K} 1$ & 1.2 & 1.0 & 6.98 & $\ldots$ & $\ldots$ & 8.49 \\
\hline V1072 Tau/TAP 35 & $\tau$ Aur & 140 & $\mathrm{~K} 1$ & 2 & 1.0 & 6.87 & $\ldots$ & 10.30 & 8.30 \\
\hline TYC $8640-2515-1 \quad \ldots \ldots \ldots$ & Sco-Cen (LCC) & 130 & & 20 & 1.1 & 6.72 & WTT & 10.77 & 8.73 \\
\hline TYC 8242-1324-1 $\ldots \ldots \ldots \ldots$ & Sco-Cen (LCC) & 130 & G0 & 16 & 1.1 & 6.72 & WTT & 10.38 & 8.14 \\
\hline TYC 8238-1462-1 $\ldots \ldots \ldots \ldots$ & Sco-Cen (LCC) & 130 & K0 & 21 & 1.1 & 6.72 & WTT & 10.10 & 8.01 \\
\hline TYC 8655-149-1 …....... & Sco-Cen (LCC) & 130 & & 19 & 1.1 & 6.72 & WTT & 10.31 & 8.37 \\
\hline TYC 8282-516-1 .......... & Sco-Cen (UCL) & 130 & & 19 & 1.1 & 6.72 & WTT & 10.68 & 8.54 \\
\hline TYC 7833-2559-1 ........ & Sco-Cen (UCL) & 130 & G6/G8 III/IV & 21 & 1.1 & 6.72 & WTT & 10.61 & 8.45 \\
\hline TYC 8694-1685-1 ......... & Sco-Cen (UCL) & 130 & & 18 & 1.1 & 6.72 & WTT & 10.21 & 8.01 \\
\hline PreibZinn 9944 ............ & U Sco & 145 & $\mathrm{~K} 2$ & 3.7 & 1.0 & 6.63 & $\ldots$ & $\ldots$ & 8.51 \\
\hline PreibZinn 9973 ............ & U Sco & 145 & K0 & 0.8 & 1.0 & 6.57 & $\ldots$ & 11.00 & 7.49 \\
\hline PreibZinn 9978 ............ & U Sco & 145 & K0 & 0.3 & 1.0 & 6.50 & $\ldots$ & 10.80 & 7.46 \\
\hline PreibZinn 9929 ............ & U Sco & 145 & $\mathrm{~K} 3 \mathrm{e}$ & 3 & 1.1 & 6.32 & $\ldots$ & 13.40 & 8.52 \\
\hline PreibZinn $9954 \quad \ldots \ldots \ldots \ldots$ & U Sco & 145 & M3 & 3 & 1.1 & 6.32 & $\ldots$ & $\ldots$ & 8.86 \\
\hline TYC 9246...71-1 .......... & Sco-Cen (LCC) & 130 & $\mathrm{G}$ & 7 & 1.2 & 6.16 & CTT & 10.54 & 7.29 \\
\hline TYC 9212-2011-1 ......... & Sco-Cen (LCC) & 130 & & 6 & 1.2 & 6.16 & WTT & 10.49 & 7.79 \\
\hline TYC 8644-340-1 .......... & Sco-Cen (LCC) & 130 & & 13 & 1.2 & 6.16 & WTT & 10.29 & 7.97 \\
\hline TYC 8645-1339-1 ........ & Sco-Cen (LCC) & 130 & & 5 & 1.2 & 6.16 & WTT & 10.82 & 7.73 \\
\hline TYC 8249-52-1 ........... & Sco-Cen (LCC) & 130 & $\mathrm{~K} 0 / \mathrm{K} 1$ & 13 & 1.2 & 6.16 & WTT & 10.48 & 8.13 \\
\hline HD $117524 \ldots \ldots \ldots \ldots \ldots$ & Sco-Cen (LCC) & 130 & G5/G6 V & 15 & 1.2 & 6.16 & WTT? & 9.84 & 7.83 \\
\hline TYC 7796-1788-1 ......... & Sco-Cen (UCL) & 130 & K5 & 13 & 1.2 & 6.16 & WTT & 10.17 & 7.88 \\
\hline TYC 7811-2909-1 ......... & Sco-Cen (UCL) & 130 & & 14 & 1.2 & 6.16 & WTT & 10.80 & 8.40 \\
\hline TYC 8283-264-1 ........... & Sco-Cen (UCL) & 130 & & 18 & 1.2 & 6.16 & WTT & 10.09 & 7.90 \\
\hline TYC 7824-1291-1 ......... & Sco-Cen (UCL) & 130 & G8 IV: & 15 & 1.2 & 6.16 & WTT & 9.80 & 7.81 \\
\hline TYC 8294-2230-1 ......... & Sco-Cen (UCL) & 130 & G7 & 17 & 1.2 & 6.16 & WTT & 10.79 & 8.71 \\
\hline TYC 7852-51-1 ........... & Sco-Cen (UCL) & 130 & F7 V & 18 & 1.2 & 6.16 & WTT & 9.05 & 7.69 \\
\hline PreibZinn $9986 \quad \ldots \ldots \ldots \ldots$ & U Sco & 145 & K0 & 1.8 & 1.1 & 6.14 & $\ldots$ & $\ldots$ & 7.76 \\
\hline PreibZinn $9983 \quad \ldots \ldots \ldots \ldots$ & U Sco & 145 & K0 & 2 & 1.1 & 6.03 & $\ldots$ & $\ldots$ & 8.51 \\
\hline PreibZinn 9971 ............ & U Sco & 145 & $\mathrm{~K} 1$ & 2.5 & 1.1 & 5.92 & $\ldots$ & 11.65 & 8.09 \\
\hline TYC 8982-3213-1 ........ & Sco-Cen (LCC) & 130 & $\mathrm{G} 1 / \mathrm{G} 2 \mathrm{~V}$ & 13 & 1.3 & 5.69 & WTT & 9.49 & 7.60 \\
\hline HD $105070 \ldots \ldots \ldots \ldots \ldots$ & Sco-Cen (LCC) & 130 & G1 V & 13 & 1.3 & 5.69 & WTT? & 8.89 & 7.31 \\
\hline TYC 8234-2856-1 ........ & Sco-Cen (LCC) & 130 & & 9 & 1.3 & 5.69 & WTT & 10.59 & 8.16 \\
\hline TYC 8633-28-1 ........... & Sco-Cen (LCC) & 130 & $\mathrm{G} 2$ & 15 & 1.3 & 5.69 & WTT & 9.49 & 7.77 \\
\hline HD $108568 \ldots \ldots \ldots \ldots \ldots$ & Sco-Cen (LCC) & 130 & G1 & 14 & 1.3 & 5.69 & WTT? & 8.89 & 7.29 \\
\hline HD $113466 \ldots \ldots \ldots \ldots \ldots$ & Sco-Cen (LCC) & 130 & G5 V: & 14 & 1.3 & 5.69 & WTT? & 9.18 & 7.36 \\
\hline TYC 7815-2029-1 ......... & Sco-Cen (UCL) & 130 & $\mathrm{~K} 0 / \mathrm{K} 1+$ & 14 & 1.3 & 5.69 & WTT? & 9.46 & 7.88 \\
\hline TYC 7833-2037-1 $\ldots \ldots \ldots$ & Sco-Cen (UCL) & 130 & $\mathrm{~K} 1$ & 10 & 1.3 & 5.69 & WTT & 11.23 & 8.73 \\
\hline TYC 7840-1280-1 ......... & Sco-Cen (UCL) & 130 & G9 & 9 & 1.3 & 5.69 & WTT & 10.57 & 8.29 \\
\hline PreibZinn 9949 ............ & U Sco & 145 & G7 & 8.5 & 1.2 & 5.53 & $\ldots$ & $\ldots$ & 8.46 \\
\hline $\mathrm{LkCa} 19 \ldots \ldots \ldots \ldots \ldots \ldots$ & $\tau$ Aur & 140 & K0 V & 2 & 1.3 & 5.49 & WTT & 10.85 & 8.15 \\
\hline PreibZinn $9925 \quad \ldots \ldots \ldots \ldots$ & U Sco & 145 & G9 & 4 & 1.2 & 5.44 & $\ldots$ & 10.99 & 8.44 \\
\hline TYC 8644-802-1 .......... & Sco-Cen (LCC) & 130 & & 6 & 1.4 & 5.28 & WTT & 10.21 & 7.66 \\
\hline HD $108611 \ldots \ldots \ldots \ldots \ldots$ & Sco-Cen (LCC) & 130 & G5 V & 10 & 1.4 & 5.28 & WTT? & 9.04 & 7.12 \\
\hline TYC $7326 \ldots 28-1 \quad \ldots \ldots \ldots \ldots$ & Sco-Cen (UCL) & 130 & $\mathrm{~K} 1$ & 7 & 1.4 & 5.28 & WTT & 10.54 & 8.12 \\
\hline HD $138995 \ldots \ldots \ldots \ldots \ldots$ & Sco-Cen (UCL) & 130 & G5 V & 10 & 1.4 & 5.28 & WTT? & 9.39 & 7.52 \\
\hline TYC 7842-250-1 .......... & Sco-Cen (UCL) & 130 & & 8 & 1.4 & 5.28 & WTT & 10.90 & 8.69 \\
\hline TYC $7333-719-1 \ldots \ldots \ldots \ldots$ & Sco-Cen (UCL) & 130 & G8 & 10 & 1.4 & 5.28 & WTT & 10.99 & 8.53 \\
\hline TYC 7853-227-1 ......... & Sco-Cen (UCL) & 130 & & 8 & 1.4 & 5.28 & WTT & 11.05 & 8.65 \\
\hline \multicolumn{10}{|c|}{ Targets Yet to Be Measured } \\
\hline GJ 803 & $\beta \mathrm{Pic}$ & 9.9 & M1 Ve & 20 & 0.4 & 242.81 & $\ldots$ & 8.81 & 4.53 \\
\hline HD $155555 \mathrm{C} \ldots \ldots \ldots \ldots \ldots$ & $\beta \mathrm{Pic}$ & 31.4 & M4.5 & 20 & 0.2 & 153.11 & $\ldots$ & 12.71 & 7.63 \\
\hline HIP $23309 \ldots \ldots \ldots \ldots \ldots$ & $\beta \mathrm{Pic}$ & 26.3 & K7 V & 20 & 0.5 & 81.25 & $\ldots$ & 10.02 & 6.24 \\
\hline HIP $3556 \ldots \ldots \ldots \ldots \ldots$ & Tuc & 45 & M1.5 & 20 & 0.3 & 71.23 & $\ldots$ & 11.91 & 7.62 \\
\hline GJ $3305 \quad \ldots \ldots \ldots \ldots \ldots \ldots$ & $\beta \mathrm{Pic}$ & 29.8 & M0.5 & 20 & 0.5 & 64.53 & $\ldots$ & 10.59 & 6.41 \\
\hline $\mathrm{CD}-641208 \ldots \ldots \ldots \ldots$ & $\beta \mathrm{Pic}$ & 29.2 & M0 & 20 & 0.6 & 54.88 & $\ldots$ & 9.54 & 6.10 \\
\hline GSC 8056-0482 & Hor & 60 & M3 Ve & 30 & 0.3 & 53.42 & $\ldots$ & 12.11 & 7.50 \\
\hline TWA $8 \mathrm{~A} \quad \ldots \ldots \ldots \ldots \ldots$ & TW Hya & 60 & M2 & 10 & 0.3 & 53.42 & $\ldots$ & $\ldots$ & 7.43 \\
\hline TWA $10 \ldots \ldots \ldots \ldots \ldots \ldots$ & TW Hya & 60 & M2.5 & 10 & 0.3 & 53.42 & $\cdots$ & $\cdots$ & 8.19 \\
\hline TWA $11 \mathrm{~B} \quad \ldots \ldots \ldots \ldots \ldots$ & TW Hya & 60 & M2.5 & 10 & 0.3 & 53.42 & $\ldots$ & 13.30 & 5.77 \\
\hline HIP $107345 \ldots \ldots \ldots \ldots \ldots$ & Tuc & 45 & M1 & 20 & 0.4 & 53.42 & $\ldots$ & 11.72 & 7.87 \\
\hline AO Men $\ldots \ldots \ldots \ldots \ldots \ldots$ & $\beta \mathrm{Pic}$ & 38.5 & $\mathrm{~K} 3$ : V: & 20 & 0.6 & 41.63 & $\ldots$ & 9.95 & 6.81 \\
\hline$\ldots \ldots \ldots \ldots$ & TW Hya & 60 & M1 & 10 & 0.4 & 40.06 & $\ldots$ & 11.06 & 6.90 \\
\hline
\end{tabular}


TANNER ET AL.

TABLE 3 (Continued)

\begin{tabular}{|c|c|c|c|c|c|c|c|c|c|}
\hline Name & Cluster & $\begin{array}{c}\text { Distance } \\
(\mathrm{pc})\end{array}$ & Spectral Type & $\begin{array}{l}\text { Age } \\
(\mathrm{Myr})\end{array}$ & $\begin{array}{c}\text { Star Mass }^{\mathrm{a}} \\
\left(M_{\odot}\right)\end{array}$ & $\begin{array}{c}\text { Signal } \\
(\mu \text { as })\end{array}$ & $\mathrm{T}$ Tauri Class & $\begin{array}{c}V \\
(\mathrm{mag})\end{array}$ & $\begin{array}{c}\text { 2MASS } K_{s} \\
\text { (mag) }\end{array}$ \\
\hline TWA $13 \ldots \ldots \ldots \ldots \ldots \ldots$ & TW Hya & 60 & M1 Ve & 10 & 0.4 & 40.06 & $\ldots$ & 11.50 & 7.49 \\
\hline HD $35850 \ldots \ldots \ldots \ldots \ldots$ & $\beta \mathrm{Pic}$ & 26.8 & F7 V & 20 & 1.0 & 35.88 & $\ldots$ & 6.30 & 4.93 \\
\hline HIP $1993 \ldots \ldots \ldots \ldots \ldots \ldots$ & Tuc & 45 & $\mathrm{~K} 7 \mathrm{~V}$ & 20 & 0.6 & 35.61 & $\ldots$ & 11.26 & 7.75 \\
\hline GSC 8499-0304 ........... & Hor & 60 & Mo Ve & 30 & 0.5 & 32.05 & $\ldots$ & 12.09 & 8.72 \\
\hline TWA $14 \ldots \ldots \ldots \ldots \ldots \ldots$ & TW Hyа & 60 & M0 & 10 & 0.5 & 32.05 & $\ldots$ & $\ldots$ & 8.50 \\
\hline TWA $18 \ldots \ldots \ldots \ldots \ldots \ldots$ & TW Hya & 60 & M0.5 & 10 & 0.5 & 32.05 & $\ldots$ & $\ldots$ & 8.85 \\
\hline HD $3221 \ldots \ldots \ldots \ldots \ldots$ & Tuc & 45 & $\mathrm{~K} 5 \mathrm{~V}$ & 20 & 0.8 & 26.71 & $\ldots$ & 9.56 & 6.53 \\
\hline GSC 8497-0995 …........ & Hor & 60 & $\mathrm{~K} 6 \mathrm{Ve}$ & 30 & 0.6 & 26.71 & $\ldots$ & 10.97 & 7.78 \\
\hline TWA $6 \quad \ldots \ldots \ldots \ldots \ldots \ldots$ & TW Hya & 60 & K7 & 10 & 0.6 & 26.71 & $\ldots$ & 12.00 & 8.04 \\
\hline TWA $1 \ldots \ldots \ldots \ldots \ldots \ldots$ & TW Hya & 60 & $\mathrm{~K} 8 \mathrm{Ve}$ & 10 & 0.6 & 26.71 & $\ldots$ & 10.92 & 7.30 \\
\hline TWA $19 B \quad \ldots \ldots \ldots \ldots \ldots \ldots$ & TW Hya & 60 & K7 & 10 & 0.6 & 26.71 & $\ldots$ & $\ldots$ & 8.28 \\
\hline V343 Nor .... & $\beta \mathrm{Pic}$ & 39.8 & $\mathrm{~K} 0 \mathrm{~V}$ & 20 & 1.0 & 24.16 & $\ldots$ & 8.14 & 5.85 \\
\hline HD $202746 \ldots \ldots \ldots \ldots \ldots$ & Tuc & 45 & $\mathrm{~K} 2 \mathrm{Vp}$ & 20 & 0.9 & 23.74 & $\ldots$ & 8.97 & 6.40 \\
\hline TWA $4 \ldots \ldots \ldots \ldots \ldots \ldots$ & TW Hya & 60 & K5 & 10 & 0.7 & 22.89 & $\ldots$ & 8.89 & 5.59 \\
\hline TWA $9 \mathrm{~A} \quad \ldots \ldots \ldots \ldots \ldots \ldots$ & TW Hya & 60 & K5 & 10 & 0.7 & 22.89 & $\ldots$ & 11.13 & 7.85 \\
\hline TWA $17 \ldots \ldots \ldots \ldots \ldots \ldots$ & TW Hya & 60 & $\mathrm{~K} 5$ & 10 & 0.7 & 22.89 & $\ldots$ & $\ldots$ & 9.01 \\
\hline HD $1466 \ldots \ldots \ldots$ & Tuc & 45 & F8/G0 V & 20 & 1.0 & 21.37 & $\ldots$ & 7.46 & 6.15 \\
\hline HD $186602 \ldots \ldots \ldots \ldots \ldots$ & Tuc & 45 & $\mathrm{~F} 7 / \mathrm{F} 8 \mathrm{~V}$ & 20 & 1.0 & 21.37 & $\ldots$ & 7.28 & 6.09 \\
\hline HD $207575 \ldots \ldots \ldots \ldots \ldots$ & Tuc & 45 & F6 V & 20 & 1.0 & 21.37 & $\ldots$ & 7.22 & 6.03 \\
\hline PPM $366328 \ldots \ldots \ldots$ & Tuc & 45 & K0 & 20 & 1.0 & 21.37 & $\ldots$ & 9.67 & 7.61 \\
\hline GSC $8047-0232 \ldots \ldots \ldots \ldots$ & Hor & 60 & $\mathrm{~K} 3 \mathrm{~V}$ & 30 & 0.8 & 20.03 & $\ldots$ & 10.87 & 8.41 \\
\hline$C D-53386 \ldots \ldots \ldots \ldots \ldots$ & Hor & 60 & $\mathrm{~K} 3 \mathrm{Ve}$ & 30 & 0.8 & 20.03 & $\ldots$ & 11.02 & 8.59 \\
\hline GSC 8862-0019 .......... & Hor & 60 & $\mathrm{~K} 4 \mathrm{Ve}$ & 30 & 0.8 & 20.03 & $\ldots$ & 11.67 & 8.91 \\
\hline$\beta$ Pic $\ldots \ldots \ldots \ldots \ldots \ldots \ldots \ldots$ & $\beta \mathrm{Pic}$ & 19.3 & A5 V & 20 & 2.5 & 19.93 & $\ldots$ & 3.85 & 3.53 \\
\hline PZ Tel $\ldots \ldots \ldots \ldots \ldots \ldots \ldots$ & $\beta \mathrm{Pic}$ & 49.7 & $\mathrm{~K} 0 \mathrm{Vp}$ & 20 & 1.0 & 19.35 & $\ldots$ & 8.43 & 6.37 \\
\hline HD $208233 \ldots \ldots \ldots \ldots \ldots$ & Tuc & 45 & G8 V & 20 & 1.2 & 17.81 & $\ldots$ & 8.90 & 6.75 \\
\hline CC Phe $\ldots \ldots \ldots \ldots \ldots \ldots \ldots$ & Hor & 60 & $\mathrm{~K} 1 \mathrm{~V}$ & 30 & 0.9 & 17.81 & $\ldots$ & 9.35 & 6.83 \\
\hline CD $-65149 \quad \ldots \ldots \ldots \ldots \ldots$ & Hor & 60 & $\mathrm{~K} 2 \mathrm{Ve}$ & 30 & 0.9 & 17.81 & $\ldots$ & 10.19 & 8.01 \\
\hline HD $172555 \ldots \ldots \ldots \ldots \ldots$ & $\beta$ Pic & 29.2 & A5 IV-V & 20 & 2.0 & 16.46 & $\ldots$ & 4.78 & 4.30 \\
\hline HD $987 \ldots \ldots \ldots \ldots \ldots \ldots$ & Tuc & 45 & G6 V & 20 & 1.3 & 16.44 & $\ldots$ & 8.76 & 6.96 \\
\hline HR $9 \ldots \ldots \ldots \ldots \ldots \ldots$ & $\beta \mathrm{Pic}$ & 39.1 & F2 IV & 20 & 1.5 & 16.39 & $\ldots$ & 6.19 & 5.24 \\
\hline CPD $-64120 \quad \ldots \ldots \ldots \ldots \ldots$ & Hor & 60 & $\mathrm{~K} 1 \mathrm{Ve}$ & 30 & 1.0 & 16.03 & $\ldots$ & 10.29 & 8.01 \\
\hline HD $202917 \ldots \ldots \ldots \ldots \ldots$ & Tuc & 45 & G5 V & 20 & 1.4 & 15.26 & $\ldots$ & 8.65 & 6.91 \\
\hline HD $195627 \ldots \ldots \ldots \ldots \ldots$ & Tuc & 45 & F1 III & 20 & 1.5 & 14.25 & $\ldots$ & 4.75 & 4.04 \\
\hline HD $195961 \ldots \ldots \ldots \ldots \ldots$ & Tuc & 45 & $\mathrm{Fm}$ & 20 & 1.5 & 14.25 & $\ldots$ & 4.86 & 3.90 \\
\hline HD $164249 \ldots \ldots \ldots \ldots \ldots$ & $\beta$ Pic & 46.9 & F5 V & 20 & 1.5 & 13.67 & $\ldots$ & 7.01 & 5.91 \\
\hline HD $207129 \ldots \ldots \ldots \ldots \ldots$ & Tuc & 45 & G0 V & 20 & 1.6 & 13.35 & $\ldots$ & 5.57 & 4.24 \\
\hline IQ Tau $\ldots \ldots \ldots \ldots \ldots \ldots \ldots$ & $\tau$ Aur & 140 & M2 & 2 & 0.5 & 13.21 & CTT & 14.50 & 7.78 \\
\hline HD 181327 & $\beta \mathrm{Pic}$ & 50.6 & F5/F6 V & 20 & 1.5 & 12.67 & $\ldots$ & 7.04 & 5.91 \\
\hline HD $178085 \ldots \ldots \ldots \ldots \ldots$ & Tuc & 45 & G0 V & 20 & 1.7 & 12.57 & $\ldots$ & 8.31 & 6.88 \\
\hline RX J012320.9-572853 ..... & HOR & 60 & G6 V & 30 & 1.3 & 12.33 & $\ldots$ & 8.53 & 6.85 \\
\hline DQ Tau $\ldots \ldots \ldots \ldots \ldots \ldots \ldots$ & $\tau$ Aur & 140 & M0 V:e & 2 & 0.6 & 12.05 & CTT & 13.66 & 7.98 \\
\hline RX J020718.6-531155 ..... & HOR & 60 & G5 V & 30 & 1.4 & 11.45 & $\ldots$ & 8.64 & 6.89 \\
\hline TWA 19A $\ldots \ldots \ldots \ldots \ldots \ldots$ & TW Hya & 60 & G3/G5 Vp & 10 & 1.4 & 11.45 & $\ldots$ & 9.07 & 7.51 \\
\hline RX J020436.7-545320 ..... & HOR & 60 & $\mathrm{~F} 2 \mathrm{~V}$ & 30 & 1.5 & 10.68 & $\ldots$ & 6.45 & 5.45 \\
\hline HD $200798 \ldots \ldots \ldots \ldots \ldots$ & Tuc & 45 & A5/A6 IV/V & 20 & 2.0 & 10.68 & $\ldots$ & 6.69 & 6.07 \\
\hline GM Tau $\ldots \ldots \ldots \ldots \ldots \ldots$ & $\tau$ Aur & 140 & M6.5 & 2 & $\ldots$ & 8.59 & $\ldots$ & $\ldots$ & 10.63 \\
\hline I0 $45251+3016 \ldots \ldots \ldots \ldots$ & $\tau$ Aur & 140 & K5 & 2 & $\ldots$ & 8.59 & $\ldots$ & 11.60 & 8.13 \\
\hline Haro $6-37 \quad \ldots \ldots \ldots \ldots \ldots$ & $\tau$ Aur & 140 & K6 & 2 & 0.8 & 8.48 & CTT & 13.42 & 7.31 \\
\hline VXR $03 \ldots \ldots \ldots \ldots \ldots$ & IC 2391 & 155 & & 53 & $\ldots$ & 7.75 & $\ldots$ & 10.95 & 14.86 \\
\hline L36 $\ldots \ldots \ldots \ldots \ldots \ldots \ldots \ldots$ & IC 2391 & 155 & F6V & 53 & $\ldots$ & 7.75 & $\ldots$ & 9.83 & 8.63 \\
\hline VXR $31 \ldots \ldots \ldots \ldots \ldots \ldots$ & IC 2391 & 155 & & 53 & $\ldots$ & 7.75 & $\ldots$ & 11.22 & 9.69 \\
\hline $\mathrm{H} 21 \ldots \ldots \ldots \ldots \ldots \ldots \ldots \ldots$ & IC 2391 & 155 & & 53 & $\ldots$ & 7.75 & $\ldots$ & 11.69 & 9.54 \\
\hline SHJM $3 \quad \ldots \ldots \ldots \ldots \ldots \ldots$ & IC 2391 & 155 & $\mathrm{~K} 3 \mathrm{e}$ & 53 & 0.8 & 7.75 & $\ldots$ & 12.63 & 9.69 \\
\hline L33 $\ldots \ldots \ldots \ldots \ldots \ldots \ldots \ldots$ & IC 2391 & 155 & F5 V & 53 & $\ldots$ & 7.75 & $\ldots$ & 9.59 & 8.36 \\
\hline H35 $\ldots \ldots \ldots \ldots \ldots \ldots \ldots \ldots$ & IC 2391 & 155 & F9 & 53 & $\ldots$ & 7.75 & $\ldots$ & 10.34 & 8.99 \\
\hline CHXR $3 \ldots \ldots \ldots \ldots \ldots \ldots$ & Cham & 140 & $\mathrm{~K} 3$ & 0.5 & 0.9 & 7.63 & WTT & 12.26 & 7.36 \\
\hline CHXR $10 \ldots \ldots \ldots \ldots \ldots$ & Cham & 140 & M0 & 2 & 0.9 & 7.63 & CTT & 11.69 & 8.20 \\
\hline ROX 3/V2245 Oph ......... & Ophiuchus & 160 & M1 & 2 & $\ldots$ & 7.51 & $\ldots$ & 13.12 & 8.78 \\
\hline HR 6070 & $\beta$ Pic & 43 & A0 V & 20 & 3.0 & 7.45 & $\ldots$ & 4.80 & 4.74 \\
\hline PreibZinn $9985 \ldots \ldots \ldots \ldots$ & U Sco & 145 & $\mathrm{~K} 2$ & 2 & 0.9 & 7.13 & $\ldots$ & $\ldots$ & 8.18 \\
\hline
\end{tabular}


TABLE 3 (Continued)

\begin{tabular}{|c|c|c|c|c|c|c|c|c|c|}
\hline Name & Cluster & $\begin{array}{l}\text { Distance } \\
(\mathrm{pc})\end{array}$ & Spectral Type & $\begin{array}{c}\text { Age } \\
(\mathrm{Myr})\end{array}$ & $\begin{array}{c}\text { Star Mass }{ }^{\mathrm{a}} \\
\left(M_{\odot}\right)\end{array}$ & $\begin{array}{c}\text { Signal } \\
(\mu \text { as })\end{array}$ & $\mathrm{T}$ Tauri Class & $\begin{array}{c}V \\
(\mathrm{mag})\end{array}$ & $\begin{array}{c}\text { 2MASS } K_{s} \\
(\mathrm{mag})\end{array}$ \\
\hline HR $136 \ldots \ldots \ldots \ldots \ldots \ldots$ & Tuc & 45 & $\mathrm{~A} 0 \mathrm{~V}$ & 20 & 3.0 & 7.12 & $\ldots$ & 5.07 & 4.99 \\
\hline HR 9062 & Tuc & 45 & A1 V & 20 & 3.0 & 7.12 & $\ldots$ & 5.00 & 4.82 \\
\hline$\ldots \ldots \ldots \ldots \ldots \ldots$ & IC 2391 & 155 & K0 & 53 & 1.0 & 6.20 & $\ldots$ & 11.86 & 9.79 \\
\hline VXR $62 \ldots \ldots \ldots \ldots \ldots$ & IC 2391 & 155 & & 53 & 1.0 & 6.20 & $\ldots$ & 11.73 & 15.03 \\
\hline VXR 67 & IC 2391 & 155 & & 53 & 1.0 & 6.20 & $\ldots$ & 11.71 & 13.61 \\
\hline VXR 16 & IC 2391 & 155 & & 53 & 1.1 & 5.64 & $\ldots$ & 11.84 & 14.58 \\
\hline VXR $72 \ldots \ldots \ldots \ldots \ldots$ & IC 2391 & 155 & G9 & 53 & 1.1 & 5.64 & $\ldots$ & 11.46 & 9.59 \\
\hline$\ldots \ldots+\ldots+\ldots \ldots$ & Tuc & 45 & B9 V & 20 & 4.0 & 5.34 & $\ldots$ & 4.36 & 4.48 \\
\hline TWA $11 \mathrm{~A} \ldots \ldots \ldots \ldots \ldots$ & TW Hya & 60 & A $0 \mathrm{~V}$ & 10 & 3.0 & 5.34 & $\ldots$ & 5.78 & 5.77 \\
\hline
\end{tabular}

a The masses presented here are either taken from the literature or are estimated using the isochrones of D'Antona \& Mazzitelli (1994).

${ }^{\mathrm{b}}$ Preibisch \& Zinnecker (1999).
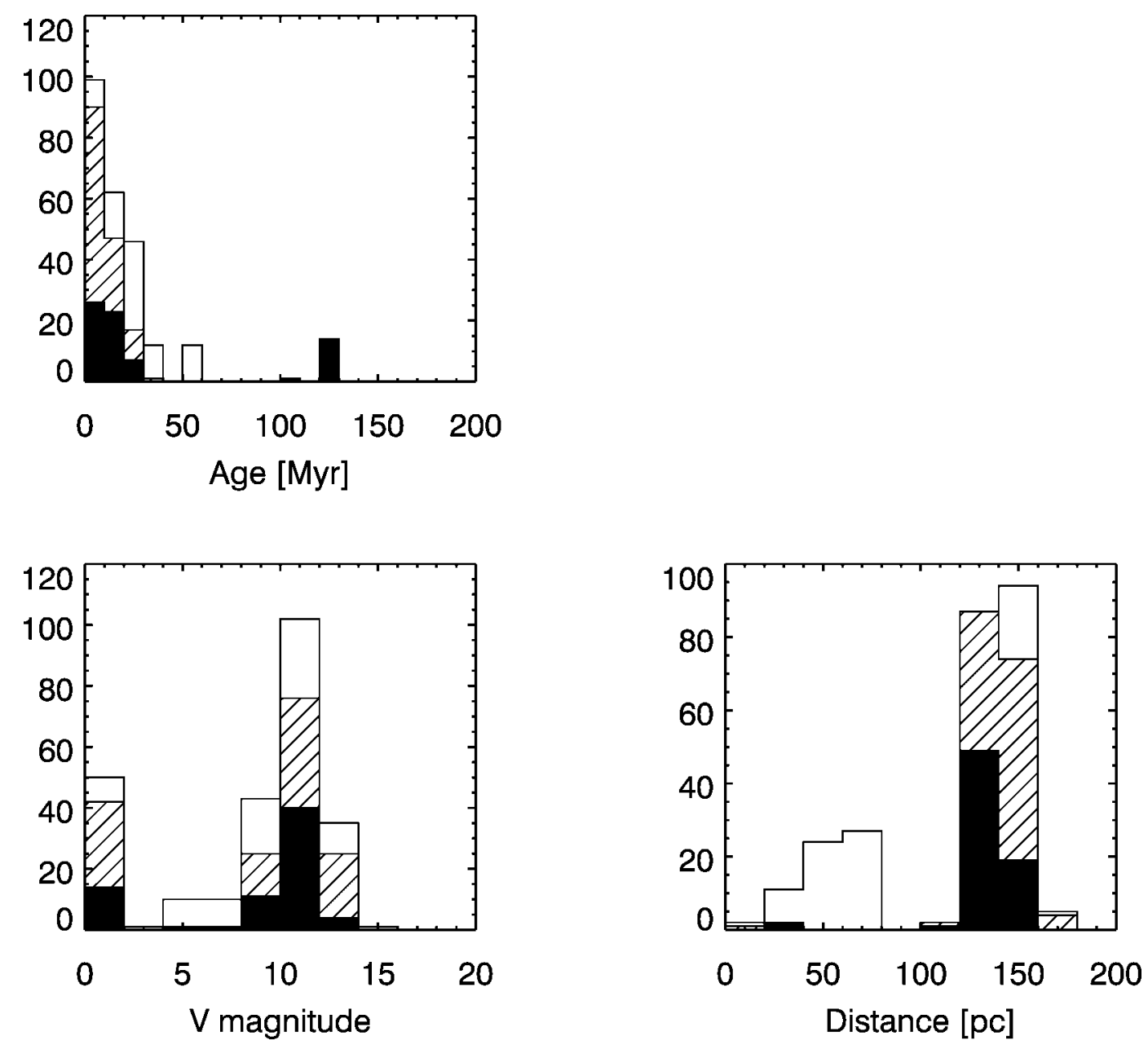

FIG. 2.-Histograms of the properties of the SIM-YSO sample. The slashed and black bars show those targets potentially eliminated and remaining, respectively, after cuts from photometry or nearby companions. The white bars represent those targets yet to be observed in the precursor surveys. 
TABLE 4

Palomar Target Sample

\begin{tabular}{|c|c|c|c|c|c|}
\hline Target & $\begin{array}{c}V \\
(\mathrm{mag})\end{array}$ & $\begin{array}{l}\text { 2MASS } K_{s} \\
\quad(\mathrm{mag})\end{array}$ & $\begin{array}{c}\text { Spectral } \\
\text { Type }\end{array}$ & $\begin{array}{l}\text { Age } \\
(\mathrm{Myr})\end{array}$ & $\begin{array}{c}\text { Distance } \\
(\mathrm{pc})\end{array}$ \\
\hline HII 1032 & 11.1 & $9.16 \pm 0.02$ & G8 & 125 & 130 \\
\hline HII $1095 \ldots \ldots \ldots$ & 11.92 & $9.67 \pm 0.02$ & K0 & 125 & 130 \\
\hline HII $1124 \ldots \ldots \ldots$ & 12.12 & $9.86 \pm 0.02$ & K1 & 125 & 130 \\
\hline HII 1136 & 12.02 & $12.14 \pm 0.02$ & G8 & 125 & 130 \\
\hline HII $1275 \ldots \ldots \ldots$ & 11.47 & $9.53 \pm 0.02$ & G8 & 125 & 130 \\
\hline HII $1309 \quad \ldots \ldots \ldots$ & 9.58 & $8.28 \pm 0.02$ & F6 & 125 & 130 \\
\hline HII $1514 \ldots \ldots \ldots$ & 10.48 & $8.95 \pm 0.02$ & G5 & 125 & 130 \\
\hline HII $1613 \ldots \ldots \ldots$ & 9.87 & $8.57 \pm 0.02$ & F8 & 125 & 130 \\
\hline HII $1794 \ldots \ldots \ldots$ & 10.2 & $8.89 \pm 0.02$ & F8 & 125 & 130 \\
\hline HII 1797 & 10.09 & $15.04 \pm 0.11$ & F9 & 125 & 130 \\
\hline HII $1856 \ldots \ldots \ldots$ & 10.2 & $8.66 \pm 0.02$ & F8 & 125 & 130 \\
\hline HII $2366 \ldots \ldots \ldots$ & 11.53 & $9.55 \pm 0.02$ & G2 & 125 & 130 \\
\hline HII $430 \ldots \ldots \ldots$ & 11.4 & $9.47 \pm 0.02$ & G8 & 125 & 130 \\
\hline HII $489 \quad \ldots \ldots \ldots$ & 10.38 & $8.87 \pm 0.02$ & F8 & 125 & 130 \\
\hline AA Tau & 12.82 & $8.05 \pm 0.02$ & K7 & 2 & 140 \\
\hline BP Tau $\ldots \ldots \ldots \ldots$ & 11.96 & $7.74 \pm 0.02$ & K7 & 2 & 140 \\
\hline DL Tau $\ldots \ldots \ldots \ldots$ & 13.55 & $7.96 \pm 0.02$ & G & 2 & 140 \\
\hline DM Tau...$\ldots \ldots$ & 13.78 & $9.52 \pm 0.02$ & K5 & 2 & 140 \\
\hline DN Tau $\ldots \ldots \ldots$ & 12.53 & $8.02 \pm 0.02$ & M0 & 2 & 140 \\
\hline DQ Tau $\ldots \ldots \ldots$ & 13.66 & $7.98 \pm 0.02$ & M0 & 2 & 140 \\
\hline DR Tau $\ldots \ldots \ldots$ & 13.6 & $6.87 \pm 0.02$ & K5 & 2 & 140 \\
\hline GK Tau $\ldots \ldots \ldots$ & 12.5 & $7.47 \pm 0.02$ & K7 & 2 & 140 \\
\hline IP Tau & 13.04 & $8.35 \pm 0.02$ & M0 & 2 & 140 \\
\hline IQ Tau & 14.5 & $7.78 \pm 0.02$ & M0.5 & 2 & 140 \\
\hline IW Tau .......... & 12.51 & $8.28 \pm 0.03$ & K7 & 2 & 140 \\
\hline LkCa 19 & 10.85 & $8.15 \pm 0.02$ & K0 & 2 & 140 \\
\hline V1072 Tau ....... & 10.3 & $8.30 \pm 0.02$ & K1 & 2 & 140 \\
\hline V830 Tau ....... & 12.21 & $8.42 \pm 0.02$ & K7 & 2 & 140 \\
\hline V836 Tau ........ & 13.13 & $8.60 \pm 0.02$ & K7 & 2 & 140 \\
\hline
\end{tabular}

paired together to act as each other's point-spread function (PSF). Two sets of known binary stars with high-quality orbital solutions (WDC 09008+4148 and WDC 23052-0742) were also observed to provide an accurate determination of the plate scale and image orientation. During these observations, each binary was placed in multiple positions over the field of view of the camera.

All the images were sky-subtracted, flat-fielded, and corrected for bad pixels. We used a number of methods for image registration prior to PSF subtraction. The best method involved using the centroid of either the waffle pattern or the "Poisson" spot (Metchev 2006). Using these methods, we were able to achieve a registration accuracy of 2.3 and 0.7 pixels, respectively, which is comparable to the accuracy achieved in a similar survey using the same instrument (Metchev \& Hillenbrand 2004). After registration, the median of each image stack was calculated to produce the final image (see Fig. 3). The images of those target stars with similar magnitudes and colors are paired and subtracted to produce a difference image intended to reduce the residual flux within 1 " of the coronagraphic spot. Prior to subtraction, each pair of images was scaled to have the same peak flux within the coronagraph halo to ensure a minimal residual flux after subtraction. Figure 4 shows the

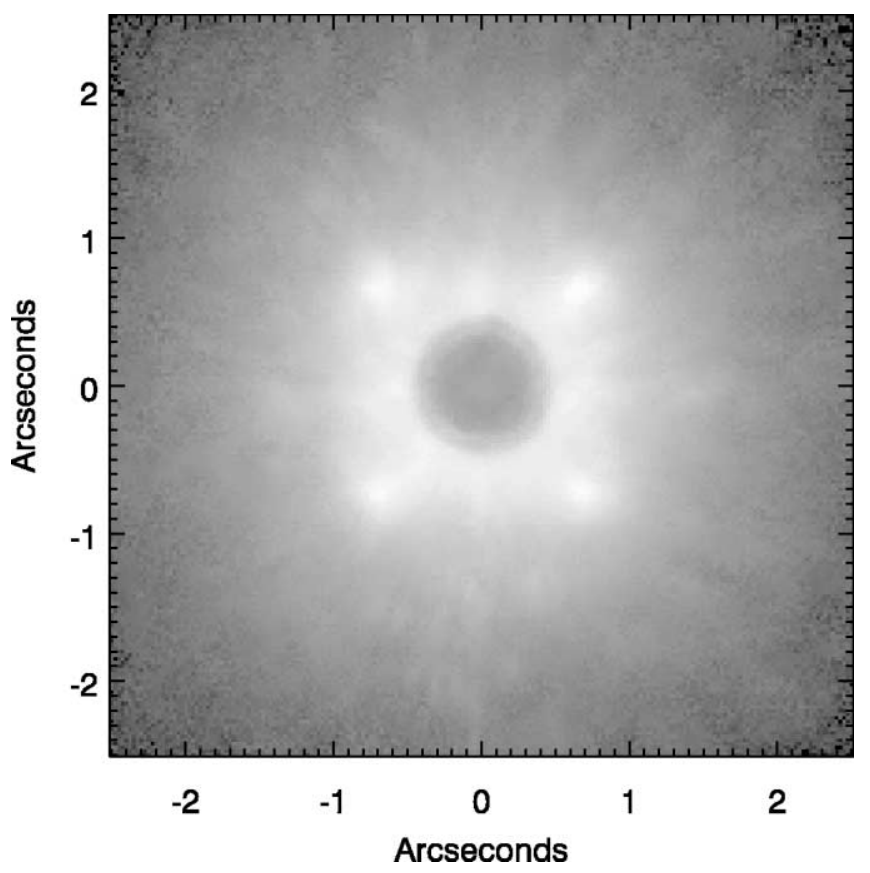

FIG. 3.-Stretched gray-scale image of LkCa 19 highlighting the Poisson spot and waffle pattern inherent in the Palomar AO+coronagraph PSF. North is up and east is to the left.

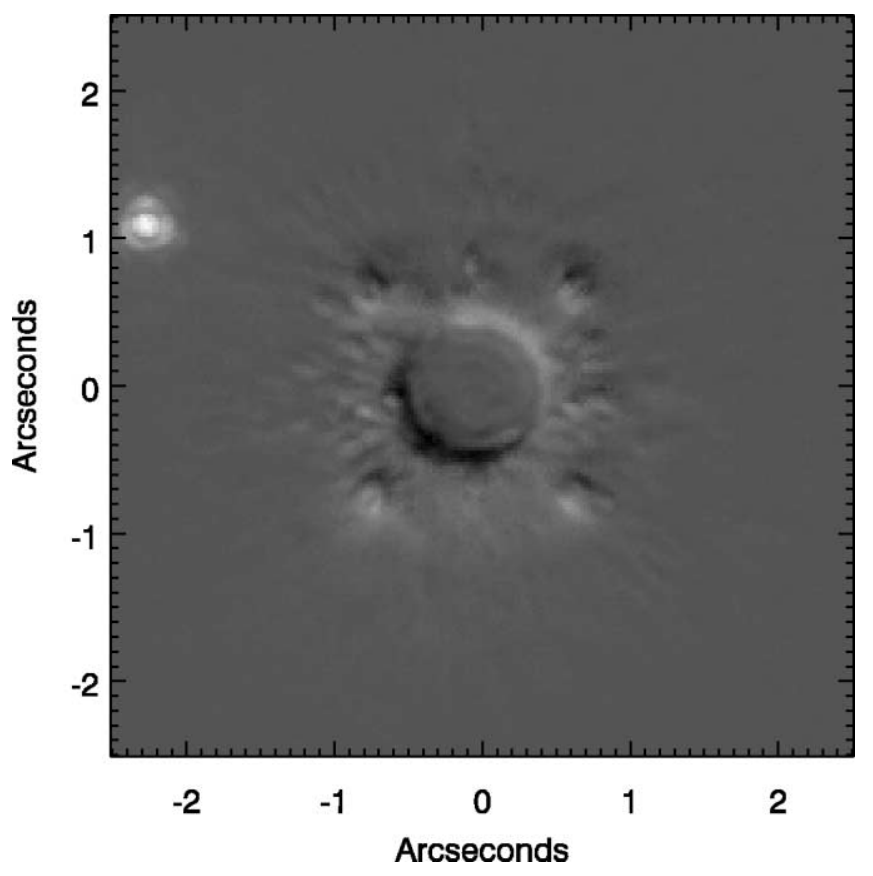

FIG. 4.-Gray-scale difference image of GK Tau and V830 Tau. The apparent companion to GK Tau is 2.4 " away but is most likely a background star, due to its blue colors and non-common proper motion. North is up and east is to the left. 
difference image of GK Tau and V830 Tau, with a bright companion candidate next to GK Tau.

A thorough visual inspection of both the median-averaged coronagraphic images and the difference images was performed to identify all potential companions. All visual companions identified in these images are listed in Table 5, along with their distance from the center of the coronagraph, the position angle, and the $K_{s}$ magnitude. The coronagraphic images have been flux-calibrated using the images taken with the primary star offset from the coronagraph while accounting for a difference in integration time and the well-defined neutral-density filter used for the off-spot images (Metchev \& Hillenbrand 2004). The magnitudes for both the primary stars in the off-spot images and the companions in the coronagraphic images are estimated from aperture photometry with an aperture of $0.9^{\prime \prime}$ and a sky annulus of $1.1^{\prime \prime}-1.4^{\prime \prime}$. The $K_{s}$-band magnitudes of the primaries were taken from the Two Micron All Sky Survey (2MASS; Cutri et al. 2003). Since many of the target stars are variable in the optical, the near-infrared photometric calibration may be uncertain, although these stars are typically 2-3 times less variable in the infrared than in the visible (Eiroa et al. 2002).

\subsection{Results}

In Taurus and the Pleiades, 10 out of 16 of the targets $(63 \%)$ and 5 out of $14(36 \%)$, respectively, have visual companions within the $25^{\prime \prime}$ ( $\left.1750 \mathrm{AU}\right)$ field of view. Almost all of the companions lie $>2$ " away from the target, corresponding to a distance greater than $300 \mathrm{AU}$. To estimate the sensitivities of our images as a function of distance from the star, we employ "PSF planting," in which a PSF corresponding to an object of known brightness is inserted into the image to determine whether it is detectable. A PSF extracted from the off-spot image of each target is sky-subtracted, normalized, multiplied by an array of contrast values $\left(\Delta K_{s}=7.7-15.1 \mathrm{mag}\right)$, and placed at a range $\left(0^{\prime \prime}-5^{\prime \prime}\right)$ of distances from the target at random position angles. We completed 10,000 iterations of the PSFplanting algorithm to fill out the parameter space of contrast and distance from the primary star. To determine whether the planted star is detected, the image is cross-correlated with a flux-normalized PSF. The correlation values are binned according to the distance of the PSF from the star, in increments of 0.1". For each distance bin, we estimate the minimum PSF intensities that resulted in a correlation value of 0.9 or higher. The intensities are converted into magnitudes using the flux calibration from the off-spot image and the 2MASS $K_{s}$ magnitude of the star. Figure 5 plots the largest $K_{s}$ magnitude difference between the target star and planted PSF with a correlation of 0.9 as a function of distance from the star for all targets with calibration data. Table 6 lists the values of the faintest detectable $K_{s}$ magnitudes at $0.5^{\prime \prime}, 1^{\prime \prime}, 2^{\prime \prime}, 5^{\prime \prime}$, and $9^{\prime \prime}$. On average, we were able to detect sources with a magnitude con-
TABLE 5

\begin{tabular}{|c|c|c|c|}
\hline Target & $\begin{array}{c}\text { Separation } \\
\text { (arcsec) }\end{array}$ & $\begin{array}{l}\text { P.A. } \\
\text { (deg) }\end{array}$ & $\begin{array}{c}K_{s} \\
(\mathrm{mag})\end{array}$ \\
\hline HII $1032 \ldots \ldots$. & 12.7 & 45.0 & 16.0 \\
\hline HII 1309 & 11.2 & -27.2 & 15.0 \\
\hline HII 1309 & 12.1 & 108.8 & 15.2 \\
\hline HII $1797 \ldots \ldots$. & 6.6 & -8.8 & 15.7 \\
\hline HII $489 \ldots \ldots .$. & 13.4 & 144.4 & 17.1 \\
\hline AA Tau $\ldots . . . .$. & 5.9 & 98.6 & 15.2 \\
\hline BP Tau ......... & 3.1 & -83.6 & 14.0 \\
\hline ВР Таu $\ldots \ldots \ldots$ & 5.6 & 8.0 & 15.0 \\
\hline DL Tau & 12.8 & 22.7 & 13.4 \\
\hline DL Tau . & 8.5 & 61.8 & 14.5 \\
\hline DL Tau . & 16.7 & 134.8 & 14.8 \\
\hline DL Tau .. & 11.9 & 15.0 & 15.4 \\
\hline DQ Tau ... & 7.3 & 149.6 & 15.3 \\
\hline GI Tau $\ldots . . . . .$. & 8.3 & 1.7 & 14.6 \\
\hline GK Tau $\ldots . . . .$. & 2.4 & 62.1 & 12.1 \\
\hline IP Tau $\ldots . . . .$. & 3.7 & 124.3 & 15.1 \\
\hline IQ Tau $\ldots . . . . .$. & 9.8 & 165.5 & 14.9 \\
\hline IQ Tau $\ldots . . . . .$. & 13.9 & -64.6 & 15.3 \\
\hline IQ Tau $\ldots . . . . .$. & 10.7 & -35.9 & 13.4 \\
\hline IQ Tau $\ldots \ldots \ldots$ & 10.2 & -33.9 & 13.6 \\
\hline LkCa $19 \ldots \ldots$ & 4.3 & -77.4 & 16.2 \\
\hline LkCa $19 \ldots \ldots$ & 11.8 & -36.2 & 13.9 \\
\hline V830 Tau ...... & 7.1 & -52.3 & 17.0 \\
\hline V830 Tau ... & 11.2 & 58.3 & 15.8 \\
\hline V830 Tau ....... & 11.8 & 100.3 & 17.5 \\
\hline V830 Таu ...... & 7.8 & 146.1 & 17.7 \\
\hline
\end{tabular}

trast of $\Delta K_{s}=4-7 \mathrm{mag}$ at $2^{\prime \prime}$ and $\Delta K_{s} \sim 8-10$ at $5^{\prime \prime}$. The range of image contrasts is due primarily to variations in seeing conditions throughout the night, since all the targets had similar magnitudes and integration times.

Calibration binaries were used to estimate the plate scale of the PHARO camera. For the 2004 October data, we adopt a plate scale of $25.11 \pm 0.04$ mas pixel ${ }^{-1}$, estimated from three different binary stars (WDS 09006+4147, WDS 18055+0230, and WDS $20467+1607)$ that were observed very close to our observations, using the same instrument (2004 October 4-5; Metchev 2006). For the 2005 data, we estimate a plate scale of $25.21 \pm 0.36 \mathrm{mas} \mathrm{pixel}^{-1}$. This plate scale and its uncertainty comes from the average and standard deviation of the separation of one binary (WDS 09006+4147) placed in multiple positions across the field of view after correction for the known distortion in the camera.

To estimate the position of the occulted target star in these images, we use the waffle pattern inherent to every PSF (see Fig. 3). Each waffle pattern consists of four points in a box pattern around the star. The center of the coronagraphic PSF is determined from the intersection of the two diagonal lines fitted to the centroid positions of the four peaks in the waffle pattern. Using this method, we are able to determine the position of the star to within 0.35 pixels, estimated from the standard deviation of the stellar position in a stack of subframes. 


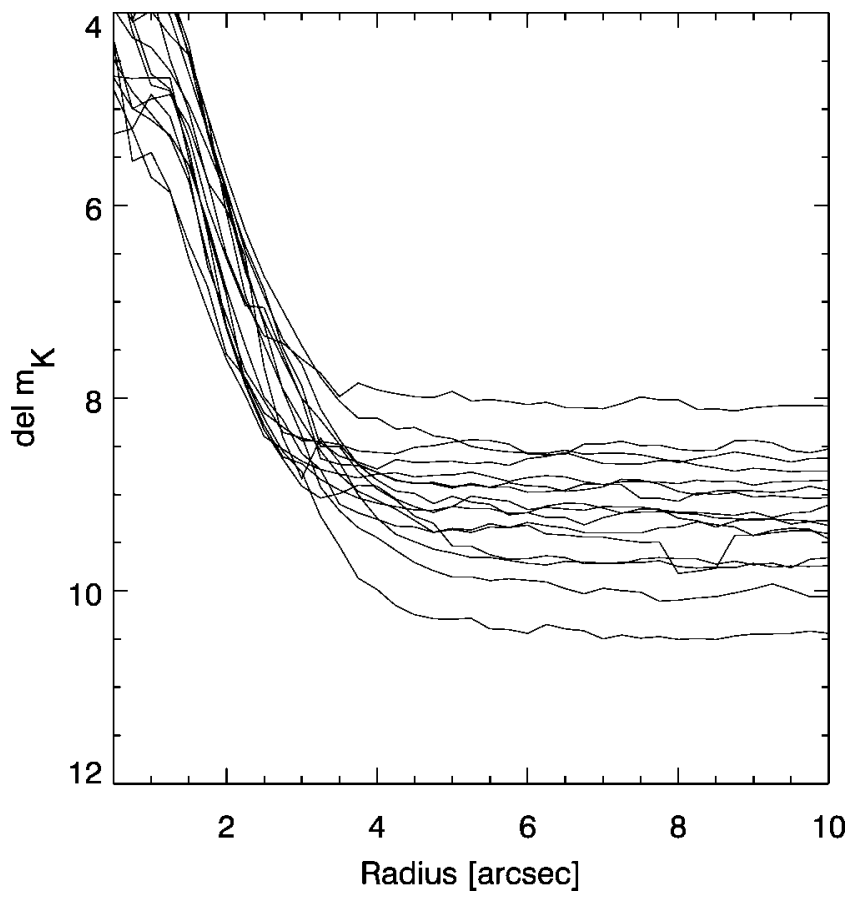

FIG. 5.-Plot of the contrast in $\Delta K_{s}$ magnitude detectable in the PHARO images as a function of separation from the target.

The positions of the companion candidates are estimated from their centroids and have errors of $0.5-1$ pixel, depending on the brightness of the companion, seeing conditions, and telescope drift. The pixel positions for all primaries and their companions are corrected for the distortion determined for the PHARO camera (Metchev 2006). The errors in the stellar position and PHARO pixel scale are propagated into the error of the offsets of the companion candidates from their primaries.

Figure 6 plots the offset in R.A. and decl. of companion candidates to BP Tau, IP Tau, GK Tau, and LkCa 19. These four sources all have objects within at least 4.5" (675 AU). While the probability of these companion candidates being background sources goes up with their separation from the target star, the discovery of a number of brown dwarf companions at wide separations ( $>200 \mathrm{AU}$ ) makes these companion candidates worth investigating further. The crosses denote the positional offsets at the observing epochs of the Palomar data (2004, 2005), in addition to data taken from Hubble Space Telescope (HST) WFPC2 and NICMOS data when available. The HST data were collected in 1999 as part of a program to detect faint debris disks (Krist et al. 2000) and therefore provide a long time baseline for the determination of common proper motion. In most cases, the target star in the WFPC2 data is saturated, and the position of the star is estimated from the intersection of the diffraction spikes. The size of the crosses denotes the $3 \sigma$ positional uncertainties $(1 \sigma \sim 12$ mas for the Palomar data, and $1 \sigma \sim 3$ mas for the WFPC2 data; J. Krist 2006, private communication). The curvy solid lines depict the
TABLE 6

Palomar Imaging Sensitivities ( $K_{s}$ Magnitude)

\begin{tabular}{|c|c|c|c|c|c|c|}
\hline Target & $\begin{array}{l}T_{\text {int }} \\
\text { (s) }\end{array}$ & $0.5^{\prime \prime}$ & $1^{\prime \prime}$ & $2^{\prime \prime}$ & $5^{\prime \prime}$ & $9^{\prime \prime}$ \\
\hline HII 1032 & 1200 & 12.84 & 13.55 & 15.91 & 18.01 & 18.17 \\
\hline HII $1136 \ldots \ldots$ & 1200 & 14.26 & 14.99 & 16.74 & 17.86 & 17.65 \\
\hline HII 1797 & 1200 & 12.17 & 12.04 & 14.53 & 17.19 & 17.28 \\
\hline HII $1794 \ldots \ldots \ldots$ & 1200 & 12.18 & 12.53 & 14.70 & 17.78 & 17.87 \\
\hline HII $489 \quad \ldots \ldots \ldots$ & 1200 & 14.68 & 13.93 & 16.20 & 17.57 & 17.76 \\
\hline AA Tau & 1200 & 13.31 & 11.44 & 15.20 & 17.16 & 17.36 \\
\hline BP Tau & 1200 & 11.08 & 11.65 & 14.26 & 17.41 & 17.57 \\
\hline CI Tau & 1200 & 12.02 & 12.10 & 13.68 & 17.67 & 17.67 \\
\hline DL Tau & 1200 & 12.51 & 13.17 & 15.24 & 17.22 & 17.20 \\
\hline DN Tau & 900 & 12.80 & 10.91 & 14.13 & 17.13 & 17.18 \\
\hline DQ Tau & 1200 & 12.84 & 13.24 & 15.01 & 17.49 & 17.39 \\
\hline GK Tau & 1200 & 11.58 & 11.22 & 12.90 & 16.94 & 17.15 \\
\hline GI Tau & 1200 & 12.00 & 12.25 & 14.16 & 17.26 & 17.34 \\
\hline IP Tau & 1200 & 13.86 & 13.00 & 15.78 & 17.04 & 17.11 \\
\hline IQ Tau ......... & 1200 & 12.27 & 13.18 & 14.93 & 16.82 & 16.93 \\
\hline LkCa $19 \ldots \ldots$ & 900 & 12.46 & 12.55 & 14.93 & 17.97 & 18.22 \\
\hline V830 Tau ........ & 1200 & 13.59 & 14.85 & 17.09 & 20.15 & 20.36 \\
\hline
\end{tabular}

changes in the offsets expected if the companion were a stationary background object. The dotted lines represent the errors in the published proper motions (Frink et al. 1997). If the companions are associated with the target stars, the offsets will coincide with one another at all epochs, since the objects will share the same space motions. Table 7 lists the reduced $\chi^{2}$ values estimated from the positional data and uncertainties. Two hypotheses are tested: common proper motion and non-common proper motion. In the first scenario, the $\chi^{2}$ is derived from the assumption that all the data points should lie on top of the first epoch (1999) data point. For the second scenario, the $\chi^{2}$ is derived from the assumption that the data points should lie on the vector produced by the change in the offset between the science target and stationary background star. The uncertainties for this case include both the positional uncertainties and the uncertainty in the proper motion of the T Tauri star (Frink et al. 1997). An unmodeled source of uncertainty that may inflate the $\chi^{2}$ values in the second scenario is the unknown proper motion of the companions. Based on this analysis, we conclude that there is evidence for common proper motion for the companion to GK Tau, evidence for non-common proper motion for the companion to BP Tau, and ambiguous evidence for either scenario for IP Tau and LkCa 19. These last two sources would benefit from more accurate positional data and a longer time baseline. If the GK Tau companion is truly a physical companion, then based on its $K_{s}$ magnitude, it would be roughly a M2-3 star with a mass of $\sim 0.5 M_{\odot}$.

We have begun a similar high-contrast imaging survey of the SIM-YSO targets in the Upper Sco subgroup of the ScoCen association (1-2 Myr, $145 \mathrm{pc}$ ), using the NAOS-CONICA (NACO) camera on the VLT. The NACO camera has been used to discover a number of low-mass companions, including 2MASSW J1207334-393254 and GQ Lupi (Neuhäuser et al. 2005; Chauvin et al. 2005). It has a pixel scale of 27 mas 

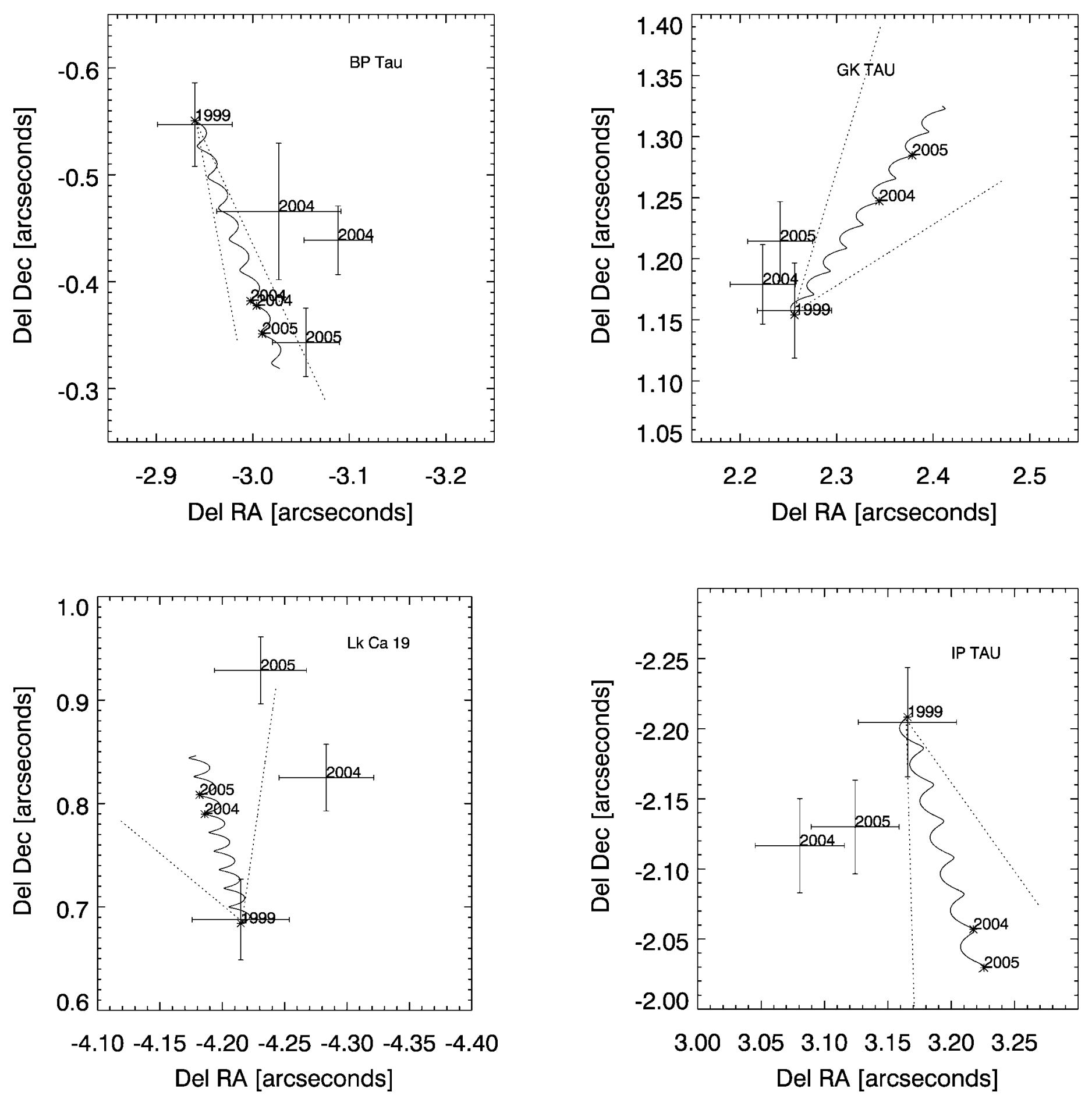

FIG. 6.-Plot of the offset in R.A. and decl. between BP Tau, GK Tau, IP Tau, and LkCa 19 and their companion candidates. The WFPC2 data point taken in 1999 January is used as the initial data point. Each measured offset is noted with a cross and an epoch label. The curvy solid line shows the expected motion of the star, assuming measured proper motions from Frink et al. (1997). The expected offset of the companion if it were a steady background object is labeled on the proper motion curve, with epoch values $(2004,2005)$. Table 6 lists the reduced $\chi^{2}$ values associated with fits to the data points, which assume the companion candidates has common and non-common proper motion with their primary stars. 
TABLE 7

Reduced $\chi^{2}$ VAlues From COMmon AND Non-COMmon Proper Motion Fits

\begin{tabular}{ccc}
\hline \hline Target & Reduced $\chi_{\text {Common PM }}^{2}$ & Reduced $\chi_{\text {Non-common PM }}^{2}$ \\
\hline BP Tau $\ldots \ldots \ldots$ & 26 & 2.5 \\
GK Tau $\ldots \ldots$. & 1.4 & 4.1 \\
IP Tau $\ldots \ldots \ldots$ & 4.2 & 4.4 \\
LkCa $19 \ldots \ldots$ & 43 & 6.3 \\
\hline
\end{tabular}

pixel $^{-1}$ and a field of view (FOV) of $28^{\prime \prime}$, with the potential of achieving $\Delta K_{s}$ sensitivities of 10 at $1^{\prime \prime}$ (Chauvin et al. 2004). So far, we have collected data on 20 targets, with a number of them having companion candidates within $1^{\prime \prime}$. We will collect second-epoch observations for these sources at a later date.

\subsection{Comparison to Other Surveys}

There have been a few other ground $\mathrm{AO}$ and space-based surveys for low-mass companions to young stars (1-200 Myr) that have found a few brown dwarfs with separations between 75 and $1000 \mathrm{AU}$. These surveys had sample sizes ranging from 30 to 100 targets, with two surveys finding two to three brown dwarfs when targeting stars in nearby associations (Metchev 2006; Lowrance et al. 2005), and one survey finding no brown dwarfs when targeting X-ray-selected T Tauri stars in the Chamaeleon and Sco-Cen OB associations (Brandner et al. 2000). Metchev (2006) has estimated a completeness-corrected percentage of brown dwarfs at $\sim 7 \% \pm 3 \%(1 \sigma$ confidence) around F5-K5 stars with an age and separation range of 3-500 Myr and 30-1600 AU, respectively. Therefore, our finding of no very low-mass objects in a survey of 30 stars is consistent with previous programs, given the stated uncertainties. Whether there is a significant dearth of brown dwarfs or planetary-mass objects around young stars still requires a larger sample of targets with similar sensitivities to allow for a direct comparison of detection statistics.

Stellar multiplicity surveys of star formation regions such as Taurus and the Pleiades have revealed binary companion fractions of 60\% (over 20-500 AU) and 30\% (over 1-900 AU), respectively (Ghez et al. 1993; Bouvier et al. 1997). However, we used these and other surveys mentioned previously (see $\S 2$ ) to remove stars with stellar companions within $2^{\prime \prime}$ in defining our initial sample. Thus, it is not surprising that our survey did not add to the statistics for stellar multiplicity in these clusters.

\subsection{Speckle Imaging and Additional AO Imaging of the Taurus Sample}

To complement the adaptive optics survey at Palomar, a number of the targets in the sample have been observed using high-resolution imaging techniques at the Keck Observatory. The purpose of this project was to look for companions at separations too close to be resolved at Palomar, and too wide to be detected via spectroscopic techniques. A summary of the results of this portion of the project is given in Table 8 .

In total, 17 objects in Taurus, TW Hydrae, or AB Doradus were surveyed at Keck. Three of these were imaged using speckle interferometry at the $K$ band $(2.2 \mu \mathrm{m})$ on Keck I, and 14 were observed using adaptive optics and the $K^{\prime}(2.3 \mu \mathrm{m})$ or $L^{\prime}$ band $(3.5 \mu \mathrm{m})$ on Keck II. The dates of these observations are given in Table 8, along with the total exposure time on each target. For details on the data reduction and analysis of both the speckle and the AO data taken at Keck, please see Konopacky et al. (2007).

For all 17 sources, no companions were found to within $0.05^{\prime \prime}$. For each source, we estimated our sensitivity to companions by finding the limiting detectable flux ratio with respect

TABLE 8

Keck Imaging Sensitivities

\begin{tabular}{|c|c|c|c|c|c|c|c|c|}
\hline \multirow[b]{2}{*}{ ОВЈЕст } & \multirow[b]{2}{*}{ DAte of OBS. } & \multirow{2}{*}{$\begin{array}{c}\text { MethoD } \\
(\mathrm{Sp} . \text { or AO) }\end{array}$} & \multirow{2}{*}{$\begin{array}{l}\text { Total Exp. } \\
\text { INT. TIME } \\
\text { (s) }\end{array}$} & \multicolumn{3}{|c|}{$\Delta K$ Limit } & \multirow{2}{*}{$\begin{array}{c}\text { Mass LIMIT }\left(M_{\odot}\right) \\
0.05^{\prime \prime}\end{array}$} & \multirow[b]{2}{*}{ BAND } \\
\hline & & & & $0.05^{\prime \prime}$ & $0.1^{\prime \prime}$ & $\geq 0.5^{\prime \prime}$ & & \\
\hline TWA 23 & 2005 May 27 & Sp. & 78.1 & 2.9 & 3.7 & 6.9 & 0.10 & $K$ \\
\hline TYC 7660-0283 ..... & 2005 May 27 & Sp. & 78.1 & 3.9 & 4.1 & 6.5 & 0.08 & $K$ \\
\hline GM Tau $\ldots \ldots \ldots \ldots$ & 1997 Oct 12 & Sp. & 156.2 & 3.9 & 3.9 & 6.5 & $\lesssim 0.02$ & $K$ \\
\hline Anon $1 \ldots \ldots$ & 2005 Dec 12 & $\mathrm{AO}$ & 120 & 3.8 & 4.4 & 6.2 & 0.06 & $K$ \\
\hline BP Таu $\ldots \ldots \ldots \ldots \ldots$ & 2005 Dec 12 & $\mathrm{AO}$ & 120 & 5.5 & 4.3 & 6.2 & 0.03 & $K$ \\
\hline DG Tau $\ldots \ldots \ldots \ldots \ldots$ & 2005 Dec 12 & $\mathrm{AO}$ & 120 & 5.8 & 3.5 & 5.9 & 0.02 & $K$ \\
\hline HD $283572 \ldots$ & 2005 Dec 12 & $\mathrm{AO}$ & 54.3 & 4.6 & 4.8 & 6.3 & 0.03 & $K$ \\
\hline IP Tau $\ldots .$. & 2005 Dec 12 & $\mathrm{AO}$ & 120 & 3.7 & 4.5 & 5.3 & 0.06 & $K$ \\
\hline IQ Tau $\ldots . .$. & 2005 Dec 12 & $\mathrm{AO}$ & 120 & 5.0 & 4.7 & 5.9 & 0.03 & $K$ \\
\hline V1072 Tau ..... & 2005 Dec 12 & $\mathrm{AO}$ & 120 & 5.1 & 5.5 & 6.5 & 0.03 & $K$ \\
\hline DN Tau $\ldots \ldots \ldots \ldots \ldots$ & 2005 Dec 12 & $\mathrm{AO}$ & 120 & 4.7 & 5.1 & 6.2 & 0.03 & $K$ \\
\hline V830 Tau $\ldots \ldots \ldots \ldots$ & 2005 Dec 12 & $\mathrm{AO}$ & 60 & 2.9 & 3.9 & 5.8 & 0.15 & $K$ \\
\hline DR Tau $\ldots \ldots \ldots \ldots \ldots$ & 2005 Dec 12 & $\mathrm{AO}$ & 54.3 & 3.4 & 3.9 & 6.6 & 0.14 & $K$ \\
\hline HIP $113579 \quad \ldots \ldots \ldots$ & 2005 Jul 16 & $\mathrm{AO}$ & 3.0 & 3.7 & 3.5 & 5.7 & 0.24 & $L$ \\
\hline HIP $113597 \quad \ldots \ldots \ldots$ & 2005 Jul 16 & $\mathrm{AO}$ & 6.0 & 3.0 & 5.1 & 5.7 & 0.12 & $L$ \\
\hline HIP $114066 \quad \ldots \ldots \ldots$ & 2005 Jul 16 & $\mathrm{AO}$ & 6.0 & 2.9 & 3.0 & 5.3 & 0.09 & $L$ \\
\hline HIP $115162 \ldots \ldots \ldots$ & 2005 Jul 16 & $\mathrm{AO}$ & 6.0 & 3.0 & 4.9 & 5.2 & 0.39 & $L$ \\
\hline
\end{tabular}


TABLE 9

KeCK INTERFEROMETRY Results

\begin{tabular}{|c|c|c|c|c|c|}
\hline Target & $K$ Mag. & No. of Ints. & Avg. $V^{2}$ & Avg. $\sigma$ & Calibrators \\
\hline DN Tau & 8.0 & 1 & 1.02 & 0.09 & HD 283444, HD 283886 \\
\hline V830 $\mathrm{Tau}^{\mathrm{a}}$ & 8.4 & 1 & 0.85 & 0.10 & HD 283668, HD 282230, HD 29334 \\
\hline V830 $\mathrm{Tau}^{\mathrm{b}}$ & 8.4 & 1 & 0.89 & 0.07 & HD 283668, HD 282230, HD 29334 \\
\hline V830 Tau . & 8.4 & 1 & 1.08 & 0.13 & HD 283444, HD 283886 \\
\hline V1171 Tau & 9.2 & 2 & 0.98 & 0.05 & HD 24132, HD 23289, HD 284316 \\
\hline V1072 Tau . & 8.3 & 1 & 1.13 & 0.10 & HIP 19757, HD 285816 \\
\hline V1075 Tau ........ & 8.9 & 1 & 1.08 & 0.10 & HIP 19757, HD 285816 \\
\hline HD $282973 \ldots \ldots$. & 8.6 & 2 & 0.97 & 0.06 & HD 24132, HD 23289, HD 284316 \\
\hline HD $282971 \ldots \ldots$ & 8.7 & 2 & 0.99 & 0.06 & HD 24132, HD 23289, HD 284316 \\
\hline HD $23584 \ldots \ldots$. & 8.3 & 2 & 0.98 & 0.06 & HD 24132, HD 23289, HD 284316 \\
\hline
\end{tabular}

${ }^{a}$ Observation from 2003 October 16.

${ }^{\text {b }}$ Observation from 2004 January 7.

to the source as a function of radius, and then using the models of Baraffe et al. (1998; $\alpha=1.0)$ to convert these flux ratio limits into mass limits for the closest radius bin of $0.5^{\prime \prime}$. In general, the speckle measurements probe regions closer than $0.05^{\prime \prime}$ with much greater sensitivity than AO, but given the combination of the two techniques for this survey, we cut off our official completeness at $0.5^{\prime \prime}$. We plan to observe the remaining targets visible from the Northern Hemisphere with either Keck AO or speckle imaging and are beginning a survey of the targets in the Southern Hemisphere using Lucky imaging, a similar observational technique at optical wavelengths (Law et al. 2006).

\section{INTERFEROMETRIC OBSERVATIONS OF STARS IN TAURUS AND THE PLEIADES}

The Keck Interferometer (KI) was used to make near-infrared, long-baseline interferometry observations of three sources in the Pleiades and five sources in Taurus. These observations are part of a long-term program to study the multiplicity fraction of these sources. In particular, the interferometer is sensitive to companions within 30 mas of the primary star and having a $K$ magnitude difference of $3 \mathrm{mag}$ or less.

The observations were taken on 2006 November 10, with KI configured in the five-channel $K$ band $(2.18 \mu \mathrm{m}$ central wavelength) mode described in Colavita et al. (2003). Calibrators were chosen to match the targets in $K$ magnitude and were reduced with the standard parameters (Colavita et al. 2003), including the ratio correction for imbalanced flux on the two paths of the interferometer. The calibrator sizes were all set to a $0.1 \pm 0.05$ mas diameter, but in all cases, the calibrator diameter did not contribute significantly to the final uncertainty. The primary KI data product is the normalized visibility amplitude squared, for which a value of 1 indicates an unresolved source. The uncertainty given is the quadrature combination of the scatter in the target measurement and the uncertainty in the system visibility (which in this case is dominated by the scatter in the calibrator measurements). Table 9 lists the targets, the number of integrations, the calibrated visibility and total uncertainty, and the calibrators used for each target. The uncer- tainty varies, due to the number of integrations and the difficulty in observing sources with fainter $V$ magnitudes.

All targets observed in this sample, except V830 Tau, are unresolved; i.e., they are consistent with a point source at this resolution. At the distance to Taurus or the Pleiades, the central star will be unresolved $(<0.1$ mas). Using the best uncertainties of 0.06 , we can place limits on the size of the emission, of 1.3 mas $(1.8 \mathrm{AU})$ in diameter $(3 \sigma)$ for a uniform disk, or on the presence of overresolved (diffuse) emission within the 50 mas FOV, of $<10 \%$ ( $3 \sigma$ ). Additional observational epochs are needed to constrain the multiplicity. V830 Tau has visibilities estimated from earlier epochs of data (2003, 2004; Akeson et al. 2005), which differ from the expected value for an unresolved source by a few $\sigma$ (see Table 9). Future observations of this star with $\mathrm{KI}$ are planned in order to determine the nature of the resolved emission.

\section{RADIAL VELOCITY VETTING}

Some of the target stars will have stellar or substellar companions that are not detectable by direct imaging (separations $<50 \mathrm{AU}$ ). We are conducting a number of radial velocity (RV) surveys of potential SIM-YSO stars to determine whether the targets have unseen companions that might complicate the astrometric detection of planetary-mass objects. For example, a $20 M_{\mathrm{J}}$ companion in a $1 \mathrm{yr}$ orbit around a $0.8 M_{\odot}$ star has an $\mathrm{RV}$ amplitude of $660 \mathrm{~m} \mathrm{~s}^{-1}$. At $140 \mathrm{pc}$, this object would produce an astrometric signature of $160 \mu$ as that would swamp the signal from any lower mass planets. Located just a few milliarcseconds from its parent star, the brown dwarf companion would be undetectable by direct noninterferometric imaging. The goal of the RV program is to achieve accuracies on the order of $<500 \mathrm{~m} \mathrm{~s}^{-1}$ over 3-4 years, depending on the $v \sin i$ and photospheric instabilities of each star, with the goal of achieving limits on stellar and substellar companions on orbits interior of $5 \mathrm{AU}$.

There are two radial velocity surveys presently being conducted on the SIM-YSO targets in both the Northern and Southern Hemispheres. The southern survey was begun in 2003 July using the echelle spectrograph $(R=25,000)$ on the CTIO 
TABLE 10

$v \sin i$ Values for Sco-Cen Targets

\begin{tabular}{|c|c|c|c|}
\hline Target & $\begin{array}{c}v \sin i \\
\left(\mathrm{~km} \mathrm{~s}^{-1}\right)\end{array}$ & Target & $\begin{array}{c}v \sin i \\
\left(\mathrm{~km} \mathrm{~s}^{-1}\right)\end{array}$ \\
\hline TYC 8648-446-1 ...... & 10 & TYC 8238-1462-1 & 25 \\
\hline TYC 8283-264-1 ...... & 10 & TYC 8654-1115-1. & 25 \\
\hline TYC 8282-516-1 ...... & 13 & TYC 8655-149-1 ...... & 25 \\
\hline TYC 8645-1339-1 ..... & 15 & TYC 9245-617-1 $\ldots \ldots$. & 25 \\
\hline TYC 9246-971-1 …... & 15 & TYC 7833-2559-1 …. & 25 \\
\hline TYC 7796-1788-1 ...... & 15 & TYC 8295-1530-1 …. & 25 \\
\hline TYC 9244-814-1 & 15 & TYC 7353-2640-1 & 25 \\
\hline TYC 7319-749-1 & 15 & TYC 8636-2515-1 & 30 \\
\hline TYC 8317-551-1 & 15 & TYC 8646-166-1 & 30 \\
\hline TYC 8242-1324-1 ..... & 18 & TYC 7310-2431-1. & 30 \\
\hline TYC 8249-52-1 ........ & 18 & TYC 8294-2230-1 ..... & 30 \\
\hline TYC 8259-689-1 ...... & 18 & TYC 7824-1291-1 ..... & 33 \\
\hline TYC 8297-1613-1 ..... & 18 & TYC 8652-1791-1 ..... & 35 \\
\hline TYC 7848-1659-1 ..... & 18 & TYC $7852-51-1 \ldots \ldots \ldots$ & 40 \\
\hline TYC $8640-2515-1 \ldots \ldots$ & 20 & TYC 8633-508-1 & 45 \\
\hline TYC 8644-340-1 ...... & 20 & TYC 8248-539-1 & 50 \\
\hline TYC 7811-2909-1 ..... & 20 & TYC 7333-1260-1 ..... & 55 \\
\hline TYC 8667-283-1 $\ldots \ldots \ldots$ & 23 & TYC 7851-1-1 $\ldots \ldots \ldots \ldots$ & 55 \\
\hline TYC 7822-158-1 $\ldots \ldots \ldots$ & 23 & TYC 7783-1908-1 ..... & 63 \\
\hline TYC 9212-2011-1 ..... & 25 & TYC $8270-2015-1 \ldots \ldots$ & 65 \\
\hline
\end{tabular}

$4 \mathrm{~m}$ telescope (PI: S. Mohanty). All 42 of the targets in this survey were in the Sco-Cen association. Only one epoch of these targets has been collected to date, because of the unfortunate retirement of this instrument on this telescope. Many of the stars in Sco-Cen turned out to be fast rotators $\left(>10 \mathrm{~km} \mathrm{~s}^{-1}\right.$; see Table 10 and Fig. 7), which is not surprising, since at 10$20 \mathrm{Myr}$ of age, there is ample time for the stars to spin-up due to stellar contraction.

The northern survey began in 2004 October and used the coudé echelle spectrograph $(R=60,000)$ on the $2.7 \mathrm{~m}$ Harlan J. Smith Telescope at McDonald Observatory (PI: L. Prato). Survey targets are located in the Pleiades and in the Taurus star-forming region. To date, 51 objects have been observed at multiple epochs, with an RV precision of $140 \mathrm{~m} \mathrm{~s}^{-1}$. This survey makes use of simultaneous $B V R$ photometry to search for correlations between RV periods and rotation that are indicative of starspot modulation rather than the presence of a low-mass companion. Initial results that include a few targets with potential companions or signs of starspots will be presented in a forthcoming publication (M. Huerta et al. 2007, in preparation; Huerta et al. 2005).

\section{PHOTOMETRIC MONITORING}

The photospheric activity that affects radial velocity and transit measurements affects astrometric measurements as well, but as we now show, at levels consistent with the secure detection of gas giant planets using SIM. From measurements of photometric variability (Herbst et al. 1994; Bouvier \& Bertout 1989; Bouvier et al. 1995) plus Doppler imaging (Strassmeier \& Rice 1998), T Tauri stars are known to have active photo-

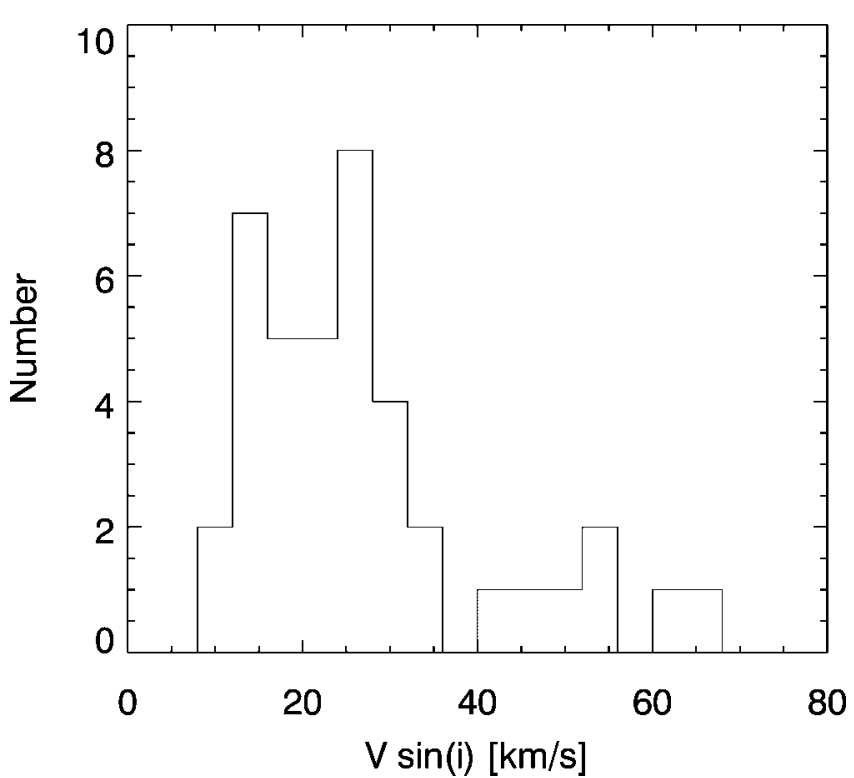

FIG. 7.-Histograms of the values of $v \sin i$ estimated from the Sco-Cen sample.

spheres, with large starspots covering significant portions of their surfaces (Schuessler et al. 1996), as well as hot spots due to infalling accreting material (Mekkaden 1998). Day-to-day changes arise primarily because of rotation, whereas monthto-month variations reflect changes in the spot sizes and their distribution across the surface. Long-term monitoring is essential, because different stars have different levels of magnetic activity, and these levels can change with time. These effects can produce large photometric variations, which can significantly shift the photocenter of a star. In the simplest approximation, a completely black starspot covering a small fraction $(\beta \ll 1)$ of a stellar hemisphere will shift the photocenter by an angle

$$
\begin{aligned}
\Delta \phi(\theta) & \sim \beta \sin \theta \cos \theta \frac{R_{\star}}{D_{\star}} \\
& =33.4 \beta \frac{R_{\star}}{R_{\odot}} \frac{140 \mathrm{pc}}{D_{\mathrm{pc}}} \sin \theta \cos \theta \mu \mathrm{as},
\end{aligned}
$$

where $R_{\star}$ is the stellar radius, $D_{*}$ is the distance to the star, and $\theta$ is the longitude of the starspot relative to the line of sight. We have assumed that the spot is on the star's equator and that the star is observed edge-on relative to its rotation axis. The shift in the photocenter $\Delta \phi(\theta)$ will increase as the spot rotates away from a face-on longitude $(\propto \sin \theta)$, following the rotation of the star as the spot shrinks in projected area $(\propto \cos \theta)$ and eventually goes behind the star. Relative to the fractional change in stellar brightness, $\Delta I / I(\theta)=\beta \cos \theta$, and averaging over $-\pi / 2<\theta<\pi / 2$, we get an rms dispersion in the location 
of the photocenter (the "astrometric jitter"), given by

$$
\begin{aligned}
\langle\Delta \phi\rangle & =\alpha\left\langle\frac{\Delta I}{I}\right\rangle \frac{R_{\star}}{D_{\star}} \\
& =1.8 \frac{R_{\star}}{R_{\odot}} \frac{140 \mathrm{pc}}{D_{\mathrm{pc}}} \frac{\Delta R(\mathrm{mag})}{0.05 \mathrm{mag}} \mu \mathrm{as},
\end{aligned}
$$

where $\alpha$ is a geometric term of order 1.1. A more careful analysis takes into account the fact that the spots are not completely black, but rather that they emit with a temperature $\sim 1000 \mathrm{~K}$ cooler than the photosphere and are located over a range of typically high latitudes, and that an ensemble of stars will be observed at random angles to the line of sight. A Monte Carlo simulation shows a linear relationship like that of equation (3), but with a smaller coefficient:

$$
\langle\Delta \phi(\text { Monte Carlo })\rangle=0.9 \frac{R_{\star}}{R_{\odot}} \frac{140 \mathrm{pc}}{D_{\mathrm{pc}}} \frac{\Delta R(\mathrm{mag})}{0.05 \mathrm{mag}} \mu \mathrm{as}
$$

For a typical T Tauri star radius of $3 R_{\odot}$ in Taurus, we see that the astrometric jitter is less than $3 \mu$ as for $R$-band variability less than or equal to $0.05 \mathrm{mag}(1 \sigma)$. Thus, the search for Jovian planets with astrometric amplitudes greater than $6 \mu$ as is possible for stars less variable than about 0.05 mag in the visible, even without a correction for jitter that may be possible using astrometric information at multiple wavelengths. Other astrophysical noise sources, such as offsets induced by the presence of nebulosity and stellar motions due to disk-induced nonaxisymmetric forces, are negligible for appropriately selected stars. Finally, it is worth noting that searching for terrestrial planets will be difficult until stars reach an age such that their photometric variability falls well below $0.01 \mathrm{mag}$ and the corresponding astrometric jitter falls below $1 \mu$ as. Even then, colordependent astrometric corrections may be needed for the most sensitive measurements.

Since young stars have active photospheres, it is important that we assess the degree of photospheric activity to determine the best targets for the program. The SIM-YSO team has two separate programs conducting photometric monitoring of the sample targets in the Northern and Southern Hemispheres. The southern targets are being observed in the $R$ band $(0.9 \mu \mathrm{m})$ at CTIO, using the SMARTS (Small and Moderate Aperture Research Telescope System) program (PI: M. Simon), which is composed of small $(0.9-1.5 \mathrm{~m})$ telescopes in the Southern Hemisphere. To date, they have observed 132 stars in the ScoCen and Upper Sco associations. Figure 8 plots the $R$ magnitudes of AA Tau and DN Tau taken with the SMARTS survey. The standard deviations of the photometry for these two sources are 0.17 and 1.5 , respectively, making the latter source a problematic SIM-YSO target.

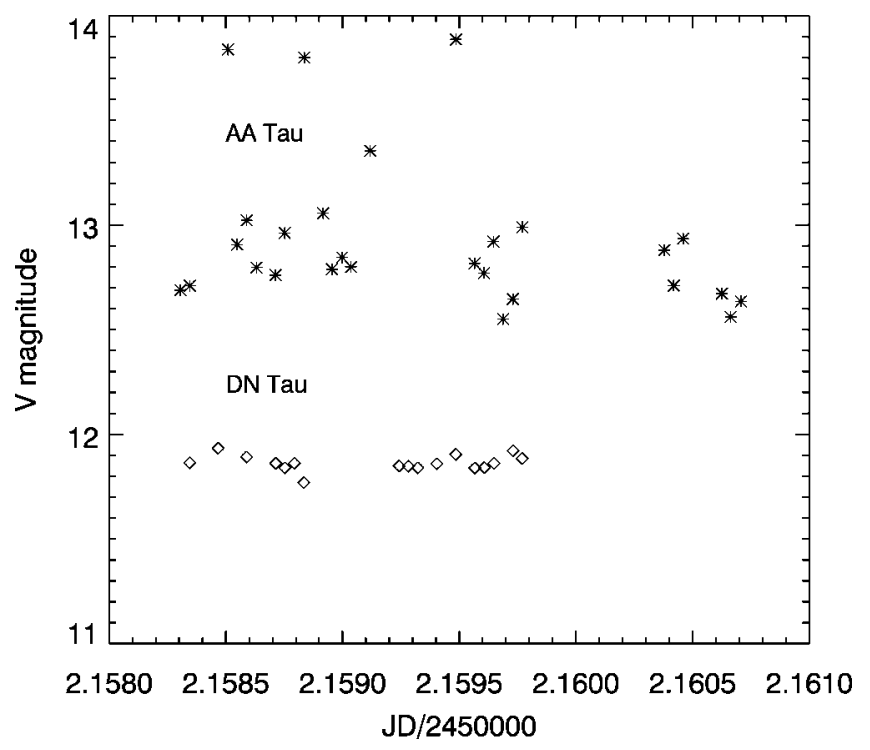

FIG. 8.-Plot of the $V$-band photometry taken for AA Tau and DN Tau. The standard deviations of the photometry for these two sources is 1.5 and 0.17 mag, respectively, making the first source a problematic SIM-YSO target.

The northern component of the SIM-YSO sample, which includes stars in Taurus and the Pleiades, has been monitored photometrically with small telescopes at the Maidanak Observatory (PI: W. Herbst; Grankin et al. 2007). Approximately 10 data points are obtained on each star during each season to sample the range of variability. The data are taken primarily in the $B, V$, and $R$ bands, with a small amount taken in the $U$ band. Forty-two stars are on the program, and about 450 individual measurements have been obtained each season.

Out of those targets observed to date in both the northern and southern samples, $33 \%$ of them have photometric variability that produces astrometric noise greater than $3 \mu$ as (or roughly $1 \sigma \sim$ $0.05 \mathrm{mag}$ ) in either the $V$ or $R$ band (see Table 11 and Fig. 9). Simultaneous monitoring of the variable stars both photometrically and with radial velocity measurements during the SIM observations might allow us to model the jitter and derive accurate astrometry. Those targets exhibiting significant amounts of photometric variability will be monitored further to assess whether they should remain in the target list or be relegated to wide-angle observations, which will be sensitive to Jupiter-mass planets farther than $1 \mathrm{AU}$ from the star.

\section{DISCUSSION}

Through a series of precursor programs to observe all of the stars in the SIM-YSO target list, it becomes clear that the ob- 
TABLE 11

Stellar Variabilities

\begin{tabular}{|c|c|c|c|c|c|c|c|}
\hline Target & Peak to Peak & $\sigma_{\text {st. dev. }}$ & Program & Target & Peak to Peak & $\sigma_{\text {st. dev. }}$ & Program \\
\hline HII 1215 & 0.024 & 0.009 & Maidanak & PreibZinn 9968 & 0.140 & 0.045 & SMARTS \\
\hline 51 Eri & 0.019 & 0.010 & Maidanak & TYC 7326-928-1 & 0.140 & 0.045 & SMARTS \\
\hline GJ 3305 & 0.020 & 0.010 & Maidanak & HD 30709 & 0.150 & 0.045 & Maidanak \\
\hline HII 996 & 0.030 & 0.010 & Maidanak & TYC 8238-1462-1 & 0.150 & 0.045 & SMARTS \\
\hline HII 489 & 0.029 & 0.012 & Maidanak & CHXR $65 \ldots \ldots \ldots \ldots$ & 0.170 & 0.045 & SMARTS \\
\hline HII 1309 & 0.037 & 0.013 & Maidanak & DM Tau .... & 0.136 & 0.046 & Maidanak \\
\hline CHXR 11 & 0.040 & 0.013 & SMARTS & PreibZinn 9959 & 0.140 & 0.046 & SMARTS \\
\hline HD 141569 & 0.056 & 0.013 & Maidanak & TYC 7319-749-1 & 0.180 & 0.046 & SMARTS \\
\hline HII $1207 \ldots$ & 0.034 & 0.014 & Maidanak & PreibZinn 9961 & 0.140 & 0.047 & SMARTS \\
\hline HII 1797 & 0.044 & 0.014 & Maidanak & PreibZinn 991 & 0.140 & 0.047 & SMARTS \\
\hline HII 1856 & 0.051 & 0.014 & Maidanak & PreibZinn 9929 & 0.170 & 0.047 & SMARTS \\
\hline HII 1095 & 0.050 & 0.015 & Maidanak & PreibZinn 9937 & 0.140 & 0.048 & SMARTS \\
\hline HII 1613 & 0.054 & 0.015 & Maidanak & TYC $8655-149-1$ & 0.140 & 0.048 & SMARTS \\
\hline HD 140374 & 0.060 & 0.015 & SMARTS & TYC 7853-227-1 & 0.140 & 0.048 & SMARTS \\
\hline HII $2366 \ldots \ldots \ldots$ & 0.041 & 0.016 & Maidanak & PreibZinn 9932 .. & 0.130 & 0.049 & SMARTS \\
\hline PreibZinn 9969 ........ & 0.050 & 0.016 & SMARTS & TYC 8283-264-1 & 0.150 & 0.049 & SMARTS \\
\hline HII $1794 \ldots \ldots \ldots \ldots \ldots$ & 0.055 & 0.016 & Maidanak & HD $149735 \ldots \ldots$ & 0.130 & 0.050 & SMARTS \\
\hline TYC 9244-814-1 ...... & 0.050 & 0.017 & SMARTS & TYC 7840-1280-1 ..... & 0.170 & 0.051 & SMARTS \\
\hline TYC 8259-689-1 ...... & 0.060 & 0.017 & SMARTS & TYC 7842-250-1 & 0.130 & 0.052 & SMARTS \\
\hline HII $430 \quad \ldots \ldots \ldots \ldots \ldots . . . .$. & 0.048 & 0.018 & Maidanak & TYC 8644-340-1 & 0.130 & 0.052 & SMARTS \\
\hline TYC 8270-2015-1 ..... & 0.050 & 0.018 & SMARTS & TYC 8694-1685-1 & 0.150 & 0.052 & SMARTS \\
\hline HII $1275 \ldots \ldots \ldots \ldots \ldots$ & 0.056 & 0.018 & Maidanak & PreibZinn 9976 & 0.160 & 0.052 & SMARTS \\
\hline TYC 7871-1282-1 ..... & 0.070 & 0.018 & SMARTS & TYC 7833-2037-1 & 0.800 & 0.052 & SMARTS \\
\hline HII 1514 & 0.059 & 0.019 & Maidanak & TYC 7852-51-1 & 0.140 & 0.053 & SMARTS \\
\hline HD 149551 & 0.060 & 0.019 & SMARTS & PreibZinn 9926 & 0.170 & 0.054 & SMARTS \\
\hline PreibZinn 9974 & 0.060 & 0.019 & SMARTS & PreibZinn 9916 & 0.170 & 0.055 & SMARTS \\
\hline TYC 8646-166-1 ........ & 0.070 & 0.019 & SMARTS & PreibZinn 9984 & & 0.055 & SMARTS \\
\hline PreibZinn $910 \quad \ldots \ldots \ldots$ & 0.070 & 0.019 & SMARTS & PreibZinn $9986 \ldots \ldots \ldots$ & 0.190 & 0.056 & SMARTS \\
\hline TYC 7848-1659-1 ..... & 0.060 & 0.020 & SMARTS & TYC 7824-1291-1 ..... & 0.190 & 0.057 & SMARTS \\
\hline CHXR $37 \ldots \ldots \ldots \ldots$ & 0.070 & 0.020 & SMARTS & PreibZinn $9923 \ldots \ldots \ldots$ & 0.150 & 0.058 & SMARTS \\
\hline CHXR $8 \ldots \ldots$ & 0.070 & 0.020 & SMARTS & TYC $8249-52-1$ & 0.150 & 0.058 & SMARTS \\
\hline PreibZinn 9919 ........ & 0.070 & 0.021 & SMARTS & TYC $8645-1339-1 \ldots \ldots$ & 0.170 & 0.058 & SMARTS \\
\hline TYC 7353-2640-1 ..... & 0.080 & 0.021 & SMARTS & HD 108611 & 0.180 & 0.058 & SMARTS \\
\hline RECX $5 \ldots \ldots \ldots \ldots$ & 0.090 & 0.021 & SMARTS & TYC 7811-2909-1 & 0.150 & 0.062 & SMARTS \\
\hline TYC 9245-617-1 ...... & 0.060 & 0.022 & SMARTS & TYC 9212-2011-1 & 0.160 & 0.062 & SMARTS \\
\hline PreibZinn 9975 & 0.070 & 0.022 & SMARTS & ROX 3 & 0.180 & 0.062 & SMARTS \\
\hline TYC 7828-2913-1 ..... & 0.070 & 0.022 & SMARTS & V1121 Oph & 0.270 & 0.063 & SMARTS \\
\hline PreibZinn 9964 ......... & 0.070 & 0.022 & SMARTS & TYC $7815-2029-1 \ldots \ldots$ & 0.220 & 0.064 & SMARTS \\
\hline TYC 8633-508-1 ...... & 0.080 & 0.022 & SMARTS & TYC 7858-830-1 & 0.170 & 0.065 & SMARTS \\
\hline TYC 8636-2515-1 ..... & 0.090 & 0.022 & SMARTS & PreibZinn 996 . & 0.190 & 0.065 & SMARTS \\
\hline PreibZinn 9981 ........ & 0.080 & 0.023 & SMARTS & PreibZinn 9963 & 0.200 & 0.066 & SMARTS \\
\hline HII $1032 \ldots$ & 0.068 & 0.024 & Maidanak & TAP 35 & 0.308 & 0.066 & Maidanak \\
\hline TYC 7310-2431-1 ..... & 0.080 & 0.024 & SMARTS & TYC 7796-1788-1 & 0.170 & 0.067 & SMARTS \\
\hline TYC 8283-2795-1 ..... & 0.080 & 0.024 & SMARTS & PreibZinn 9940 & 0.180 & 0.067 & SMARTS \\
\hline TYC 8667-283-1 ...... & 0.090 & 0.024 & SMARTS & RECX 4 & 0.200 & 0.067 & SMARTS \\
\hline TYC 7305-380-1 & 0.090 & 0.025 & SMARTS & PreibZinn 9977 & 0.160 & 0.068 & SMARTS \\
\hline TYC 7349-2191-1 ..... & 0.090 & 0.025 & SMARTS & PreibZinn 993 & 0.200 & 0.068 & SMARTS \\
\hline TYC 7310-503-1 ...... & 0.080 & 0.026 & SMARTS & CHXR $18 \mathrm{~N}$ & 0.210 & 0.068 & SMARTS \\
\hline PreibZinn 9966 ........ & 0.080 & 0.027 & SMARTS & SR4 ... & 0.200 & 0.069 & SMARTS \\
\hline PreibZinn 9982 ......... & 0.080 & 0.027 & SMARTS & TYC $7333-719-1$ & 0.240 & 0.069 & SMARTS \\
\hline TYC 8297-1613-1 ..... & 0.100 & 0.027 & SMARTS & ROX 43A ....... & 0.180 & 0.072 & SMARTS \\
\hline TYC 9231-1566-1 ..... & 0.100 & 0.027 & SMARTS & Haro 6-37 ...... & 0.230 & 0.075 & SMARTS \\
\hline PreibZinn 992 .......... & 0.090 & 0.028 & SMARTS & CHXR 66 & 0.220 & 0.076 & SMARTS \\
\hline TYC $8258-1878-1 \ldots \ldots$ & 0.090 & 0.028 & SMARTS & PreibZinn 9918 & 0.240 & 0.077 & SMARTS \\
\hline TYC $8317-551-1 \ldots \ldots$. & 0.100 & 0.028 & SMARTS & PreibZinn 9955 & 0.230 & 0.078 & SMARTS \\
\hline TYC 8652-1791-1 ..... & 0.100 & 0.029 & SMARTS & V830 Tau $\ldots \ldots \ldots \ldots \ldots$ & 0.346 & 0.078 & Maidanak \\
\hline TYC 7845-1174-1 ..... & 0.110 & 0.029 & SMARTS & PreibZinn 9970 & 0.230 & 0.079 & SMARTS \\
\hline CHXR 40 & 0.100 & 0.030 & SMARTS & TYC 8294-2230-1 & 0.230 & 0.079 & SMARTS \\
\hline PreibZinn 9913 & 0.100 & 0.030 & SMARTS & L1551-51 & 0.258 & 0.079 & Maidanak \\
\hline PreibZinn 9945 & 0.100 & 0.030 & SMARTS & V966 Cen & 0.130 & 0.080 & SMARTS \\
\hline TYC 7783-1908-1 ..... & 0.110 & 0.030 & SMARTS & PreibZinn 9911 & 0.230 & 0.081 & SMARTS \\
\hline PreibZinn 9917 ......... & 0.110 & 0.030 & SMARTS & HD $105070 \ldots$ & 0.230 & 0.083 & SMARTS \\
\hline
\end{tabular}


TABLE 11 (Continued)

\begin{tabular}{|c|c|c|c|c|c|c|c|}
\hline Target & Peak to Peak & $\sigma_{\text {st. dev. }}$ & Program & Target & Peak to Peak & $\sigma_{\text {st. dev. }}$ & Program \\
\hline TYC 8983-98-1 & 0.110 & 0.030 & SMARTS & CHXR 29 & 0.260 & 0.083 & SMARTS \\
\hline PreibZinn 9958 & 0.090 & 0.031 & SMARTS & TYC 7813-224-1 & 0.080 & 0.084 & SMARTS \\
\hline TYC 8295-1530-1 ..... & 0.100 & 0.031 & SMARTS & TYC 8982-3213-1 & 0.250 & 0.085 & SMARTS \\
\hline TYC 7822-158-1 ...... & 0.110 & 0.031 & SMARTS & IW Tau ......... & 0.238 & 0.086 & Maidanak \\
\hline TYC 7333-1260-1 ..... & 0.110 & 0.031 & SMARTS & V1056 Sco ........ & 0.230 & 0.087 & SMARTS \\
\hline V1009 Cen .............. & 0.090 & 0.032 & SMARTS & TYC 9246-971-1 & 0.240 & 0.088 & SMARTS \\
\hline PreibZinn $9939 \quad \ldots \ldots \ldots$ & 0.090 & 0.034 & SMARTS & PreibZinn 9925 & 0.250 & 0.088 & SMARTS \\
\hline HD 140421 & 0.110 & 0.034 & SMARTS & PreibZinn 9933 & 0.250 & 0.091 & SMARTS \\
\hline TYC 8263-2453-1 ..... & 0.110 & 0.034 & SMARTS & PreibZinn 9954 & 0.240 & 0.092 & SMARTS \\
\hline PreibZinn 9914 & 0.120 & 0.034 & SMARTS & PreibZinn 9949 & 0.210 & 0.093 & SMARTS \\
\hline LkCa 19 & 0.163 & 0.034 & Maidanak & TYC 8242-1324-1 & 0.320 & 0.094 & SMARTS \\
\hline TYC $8654-1115-1 \ldots \ldots$ & 0.120 & 0.035 & SMARTS & PreibZinn $9980 \quad \ldots \ldots \ldots$ & 0.260 & 0.097 & SMARTS \\
\hline TYC 8640-2515-1 ..... & 0.130 & 0.035 & SMARTS & PreibZinn 9942 & 0.290 & 0.097 & SMARTS \\
\hline PreibZinn 99-79 ....... & 0.100 & 0.036 & SMARTS & PreibZinn 9921. & 0.300 & 0.107 & SMARTS \\
\hline TYC 8683-242-1 ...... & 0.110 & 0.036 & SMARTS & HQ Tau $\ldots . .$. & 0.233 & 0.109 & Maidanak \\
\hline PreibZinn 9928 ........ & 0.110 & 0.037 & SMARTS & CI Tau & 0.298 & 0.114 & Maidanak \\
\hline PreibZinn 9960 ......... & 0.110 & 0.037 & SMARTS & IP Tau ............. & 0.362 & 0.125 & Maidanak \\
\hline PreibZinn 9967 ........ & 0.120 & 0.037 & SMARTS & PreibZinn 9915 . & 0.430 & 0.126 & SMARTS \\
\hline$\ldots \ldots+\ldots \ldots$ & 0.100 & 0.038 & Maidanak & TYC 7817-622-1 & 0.380 & 0.127 & SMARTS \\
\hline PreibZinn 9927 ... & 0.140 & 0.038 & SMARTS & BP Tau & 0.457 & 0.129 & Maidanak \\
\hline PreibZinn $9962 \ldots \ldots \ldots$ & 0.140 & 0.038 & SMARTS & UY Aur . & 0.350 & 0.130 & Maidanak \\
\hline RECX $6 \ldots \ldots \ldots$ & 0.150 & 0.038 & SMARTS & SR $10 \ldots$ & 0.370 & 0.131 & SMARTS \\
\hline DN Tau & 0.165 & 0.038 & Maidanak & DoAr 21 & 0.310 & 0.134 & SMARTS \\
\hline PreibZinn 9936 ........ & 0.110 & 0.039 & SMARTS & DG Tau $\ldots \ldots \ldots . .$. & 0.606 & 0.138 & Maidanak \\
\hline CHXR $6 \ldots \ldots \ldots \ldots$ & 0.120 & 0.039 & SMARTS & PreibZinn 9978 ........ & 0.350 & 0.139 & SMARTS \\
\hline HD 120411 & 0.120 & 0.039 & SMARTS & V836 Tau .... & 0.417 & 0.142 & Maidanak \\
\hline RECX 10 & 0.120 & 0.039 & SMARTS & HD 113466 & 0.390 & 0.149 & SMARTS \\
\hline TYC 8248-539-1 ...... & 0.120 & 0.039 & SMARTS & GK Tau $\ldots \ldots \ldots$ & 0.692 & 0.183 & Maidanak \\
\hline PreibZinn 922 ........ & 0.110 & 0.040 & SMARTS & PreibZinn 9973 ......... & 0.550 & 0.188 & SMARTS \\
\hline TYC 8319-1687-1 ..... & 0.120 & 0.040 & SMARTS & DL Tau $\ldots . .$. & 0.723 & 0.212 & Maidanak \\
\hline PreibZinn 9971 ......... & 0.140 & 0.040 & SMARTS & DH Tau . & 0.537 & 0.214 & Maidanak \\
\hline HD $138995 \quad \ldots \ldots \ldots \ldots$ & 0.150 & 0.040 & SMARTS & GI Tau & 0.597 & 0.216 & Maidanak \\
\hline L1551-55 „............ & 0.126 & 0.041 & Maidanak & TYC 8633-28-1 ........ & 0.180 & 0.226 & SMARTS \\
\hline TYC 7833-2559-1 ...... & 0.140 & 0.041 & SMARTS & HD 108568 & 0.550 & 0.233 & SMARTS \\
\hline PreibZinn 9983 ........ & 0.130 & 0.042 & SMARTS & PreibZinn 9944 & 0.670 & 0.262 & SMARTS \\
\hline TWA $19 \ldots \ldots \ldots \ldots$ & 0.140 & 0.042 & SMARTS & DR Tau $\ldots \ldots \ldots \ldots \ldots$ & 1.092 & 0.296 & Maidanak \\
\hline HD 117524 & 0.140 & 0.043 & SMARTS & AA Tau & 1.499 & 0.454 & Maidanak \\
\hline TYC 8644-802-1 ...... & 0.140 & 0.043 & SMARTS & TYC 8234-2856-1 ...... & 0.170 & 0.495 & SMARTS \\
\hline CHXR 68A ............ & 0.150 & 0.043 & SMARTS & TYC 8282-516-1 ...... & 0.130 & 0.689 & SMARTS \\
\hline TYC 7851-1-1 ......... & 0.120 & 0.044 & SMARTS & $\operatorname{RECX} 2 \ldots \ldots \ldots \ldots$ & 2.150 & 0.808 & SMARTS \\
\hline TYC 8648-446-1 ...... & 0.150 & 0.044 & SMARTS & T Cha $\ldots$. & 2.430 & 0.850 & SMARTS \\
\hline PreibZinn $9950 \ldots \ldots \ldots$ & 0.120 & 0.045 & SMARTS & HII $1136 \ldots$ & 1.841 & 1.127 & Maidanak \\
\hline
\end{tabular}

servable most affecting the viability of the targets is photometric variability. To date, $22 \%$ of the stars (33\% of those stars observed) in the target list have variability that contributes more than $3 \mu$ as of noise to the astrometric measurements. Table 3, which provides basic information on the entire SIM-YSO list, has been separated into "low variability," "high variability," and "not observed" sections, based on the degree of their variability and whether they have been observed to date. Each sublist is then ranked by the astrometric signal expected for a Jupiter-mass planet at a distance of $1 \mathrm{AU}$ from the stars (listed in the column labeled "Signal"). To date, $67 \%$ of the sample has variability data, with the remainder expected to be completed within the next year or two.

To replace those stars that might be lost to photometric variability, we will examine the literature, looking for objects in other clusters and newly classified T Tauri stars. While many new young stars are being discovered in relatively nearby moving groups (e.g., Song et al. 2003), these typically have ages of 10-25 Myr. These stars can be observed inexpensively with SIM's wide-angle mode and will probably not have problems of excessive variability. We will concentrate on finding bright enough replacement stars in the 1-5 Myr age range to avoid skewing our sample toward older stars.

\subsection{Observing Scenarios and Reference Stars}

While the nearer target stars $(d<50 \mathrm{pc})$ will have planetary astrometric signatures large enough to be detected by wideangle observations, more distant or more massive stars will have astrometric signatures on the order of $6 \mu \mathrm{as}$, requiring 


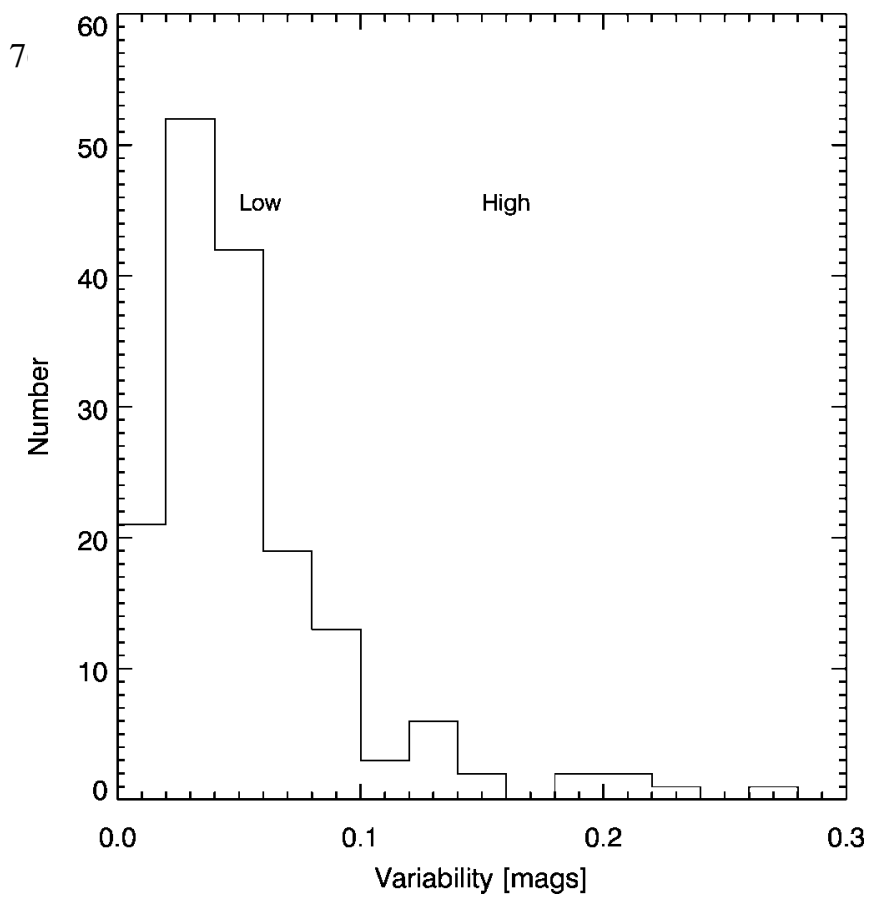

FIG. 9.-Histograms of the standard deviations of the flux variations observed in both the northern and southern photometry surveys. A deviation of $>0.05 \mathrm{mag}$ is considered too high for the SIM-YSO targets.

narrow-angle observations. Narrow-angle observations require that a set of at least three reference stars within the $\sim 1.5^{\circ}$ field of view be used for these observations. These reference stars must themselves be astrometrically stable to within $<4 \mu$ as. The best reference stars are $\mathrm{K}$ giants at distances $>500$ pc. There is an additional precursor program currently making photometric observations of the pool of reference stars that are available for every SIM-YSO target (or clusters of targets). When choosing the reference stars, we used a combined 2MASS-Tycho 2 catalog to select $\mathrm{K}$ giants based on visible-near-IR colors and on reduced proper motions (RPMs). The following selection criteria were also used to make the initial lists of reference stars: (1) separation from target $<1.25^{\circ}$, (2) $0.5<\left(J-K_{s}\right)<1.0$, (3) $1.0<\left(B_{\text {Tycho }}-V_{\text {Tycho }}\right)<1.5$, (4) $V_{\text {Tycho }}<10$, and (5) RPM $=$ $K_{s}+5 \log \mu<1$. While some of the reference stars have published spectral types, many stars do not have any spectral type at all. We have begun a program of verification of the luminosity classes of the photometrically selected sample, using the SMARTS telescopes.

Observing the stars in narrow-angle mode requires roughly 5 times more integration time than doing so in wide-angle mode, primarily due to the necessity of observing three to five reference stars. Putting together the final program will require a balance between observing fainter, more distant, and young objects in narrow-angle mode versus the brighter, closer, and older stars in wide-angle mode.

\section{CONCLUSIONS}

We have presented the results for a number of precursor programs aimed at creating a robust list of young stars to be observed as part of a key project for the SIM PlanetQuest mission. This program will detect Jupiter-mass planets at distances of $\sim 1-5$ AU from the star, thereby probing planet formation at distances comparable to where radial velocity planets are found around mature main-sequence stars. The imaging surveys did not find any stars with bright nearby companions that could pose a problem for SIM, although we did find a potential M star companion 2.4" away from GK Tau. The radial velocity surveys may have found one or two stars with closein companions. In the near future, the RV surveys will be supplemented with high spectral resolution RV surveys in the near-infrared. These observations are not as affected by the photometric variability of the star and are expected to achieve rms accuracies down to $100 \mathrm{~m}^{-1}$, thereby allowing for the detection of lower mass objects. One selection effect we will have to guard against is losing too many of the youngest stars due to large photometric variations. We will continue to supplement our target list with additional stars that meet our basic criteria, and also investigate ways to mitigate astrometric jitter using multiwavelength data from SIM itself.

We acknowledge many useful and formative discussions with other members of the SIM-YSO Science Team, including A. Boden, L. Hartmann, J. Lunine, J. Stauffer, and T. Velusamy. L. P. and M. H. thank our colleagues C. Johns-Krull, P. Hartigan, and D. Jaffe for their collaboration on the McDonald Observatory project. Based on observations obtained at the Hale Telescope, Palomar Observatory, as part of a continuing collaboration between the California Institute of Technology, the NASA Jet Propulsion Laboratory, and Cornell University. The research described in this publication was carried out at the Jet Propulsion Laboratory, California Institute of Technology, under a contract with the National Aeronautics and Space Administration. This publication makes use of data products from the Two Micron All Sky Survey, which is a joint project of the University of Massachusetts and the Infrared Processing and Analysis Center, California Institute of Technology, funded by the National Aeronautics and Space Administration and the National Science Foundation.

\section{REFERENCES}

Akeson, R. L., et al. 2005, ApJ, 635, 1173

Baraffe, I., Chabrier, G., Allard, F., \& Hauschildt, P. H. 1998, A\&A, 337, 403
Baraffe, I., Chabrier, G., Barman, T. S., Allard, F., \& Hauschildt, P. H. 2003, A\&A, 402, 701 
Beichman, C. A. 2001, in ASP Conf. Ser. 244, Young Stars Near Earth: Progress and Prospects, ed. R. Jayawardhana \& T. Greene (San Francisco: ASP), 376

Boss, A. P. 2001, ApJ, 563, 367

Bouvier, J., Rigaut, F., \& Nadeau, D. 1997, A\&A, 323, 139

Bouvier, J., Covino, E., Kovo, O., Martin, E. L., Matthews, J. M., Terranegra, L., \& Beck, S. C. 1995, A\&A, 299, 89

Bouvier, J., \& Bertout, C. 1989, A\&A, 211, 99

Brandner, W., et al. 2000, AJ, 120, 950

Burrows, A., et al. 1997, ApJ, 491, 856

Butler, R. P., et al. 2006, ApJ, 646, 505

Catanzarite, J., Shao, M., Tanner, A., Unwin, S., \& Yu, J. 2006, PASP, 118,1322

Chauvin, G., et al. 2005, A\&A, 438, L29

Chauvin, G., Lagrange, A.-M., Dumas, C., Zuckerman, B., Mouillet, D., Song, I., Beuzit, J.-L., \& Lowrance, P. 2004, A\&A, 425, L29 Colavita, M., et al. 2003, ApJ, 592, L83

Cutri, R. M., et al. 2003, The IRSA 2MASS All-Sky Point Source Catalog (NASA/IPAC Infrared Science Archive; Washington: NASA), http://irsa.ipac.caltech.edu/applications/Gator

D’Antona, F., \& Mazzitelli, I. 1994, ApJS, 90, 467

Eiroa, C., et al. 2002, A\&A, 384, 1038

Frink, S., Röser, S., Neuhäuser, R., \& Sterzik, M. F. 1997, A\&A, 325, 613

Ghez, A. M., Neugebauer, G., \& Matthews, K. 1993, AJ, 106, 2005

Grankin, K. N., Melnikov, S. Y., Bouvier, J., Herbst, W., \& Shevchenko, V. S. 2007, A\&A, 461, 183

Hayward, T. L., Brandl, B., Pirger, B., Blacken, C., Gull, G. E., Schoenwald, J., \& Houck, J. R. 2001, PASP, 113, 105

Herbst, W., Herbst, D. K., Grossman, E. J., \& Weinstein, D. 1994, AJ, 108, 1906

Huerta, M., Prato, L., Hartigan, P., Johns-Krull, C. M., \& Jaffe, D. 2005, BAAS, 37, 1267

Kenyon, S. J., Dobrzycka, D., \& Hartmann, L. 1994, AJ, 108, 1872

Konopacky, Q. M., Ghez, A. M., Rice, E. L., \& Duchene, G. 2007 , ApJ, 663, 394
Krist, J. E., Stapelfeldt, K. R., Ménard, F., Padgett, D. L., \& Burrows, C. J. 2000, ApJ, 538, 793

Ida, S., \& Lin, D. N. C. 2004, ApJ, 616, 567

Law, N. M., Hodgkin, S. T., \& Mackay, C. D. 2006, MNRAS, 368, 1917

Lin, D. 2001, in ASP Conf. Ser. 245, Astrophysical Ages and Times Scales, ed. T. von Hippel, C. Simpson, \& N. Manset (San Francisco: ASP), 90

Lowrance, P. J., et al. 2005, AJ, 130, 1845

Marcy, G. W., \& Butler, R. P. 2000, PASP, 112, 137

Mathieu, R. D., Stassun, K., Basri, G., Jensen, E. L. N., Johns-Krull, C. M., Valenti, J. A., \& Hartmann, L. W. 1997, AJ, 113, 1841

Mekkaden, M. V. 1998, A\&A, 340, 135

Metchev, S. 2006, Ph.D. Thesis, Caltech

Metchev, S. A., \& Hillenbrand, L. A. 2004, ApJ, 617, 1330

Neuhäuser, R., Guenther, E. W., Wuchterl, G., Mugrauer, M., Bedalov, A., \& Hauschildt, P. H. 2005, A\&A, 435, L13

Pan, S., Shao, M., \& Kulkarni, S. 2004, Nature, 427, 326

Preibisch, T., \& Zinnecker, H. 1999, AJ, 117, 2381

Pollack, J. B., Hubickyj, O., Bodenheimer, P., Lissauer, J. J., Podolak, M., \& Greenzweig, Y. 1996, Icarus, 124, 62

Schuessler, M., Caligari, P., Ferriz-Mas, A., Solanki, S. K., \& Stix, M. 1996, A\&A, 314, 503

Song, I., Zuckerman, B., \& Bessell, M. S. 2003, ApJ, 599, 342

Sozzetti, A., Casertano, S., Brown, R. A., \& Lattanzi, M. G. 2003, PASP, 115, 1072

Stauffer, J. R., Schild, R., Barrado y Navascues, D., Backman, D. E., Angelova, A. M., Kirkpatrick, J. D., Hambly, N., \& Vanzi, L. 1998, ApJ, 504, 805

Steffen, A. T., et al. 2001, AJ, 122, 997

Strassmeier, K. G., \& Rice, J. B. 1998, A\&A, 339, 497

Unwin, S. C. 2005, in ASP Conf. Ser. 338, Astrometry in the Age of the Next Generation of Large Telescopes, ed. P. K. Seidelmann \& A. K. B. Monet (San Francisco: ASP), 37

White, R. J., \& Ghez, A. M. 2001, ApJ, 556, 265

Wuchterl, G., \& Tscharnuter, W. M. 2003, A\&A, 398, 1081 